\title{
Open string QED meson description of the X17 particle and dark matter
}

\author{
Cheuk-Yin Wong \\ Physics Division, Oak Ridge National Laboratory, \\ Oak Ridge, Tennessee 37831, U.S.A. \\ E-mail: wongc@ornl.gov
}

ABSTRACT: As a quark and an antiquark cannot be isolated, the intrinsic motion of a composite $q \bar{q}$ system in its lowest-energy states lies predominantly in $1+1$ dimensions, as in an open string with the quark and the antiquark at its two ends. Accordingly, we study the lowest-energy states of an open string $q \bar{q}$ system in QCD and QED in $1+1$ dimensions. We show that $\pi^{0}, \eta$, and $\eta^{\prime}$ can be adequately described as open string $q \bar{q}$ QCD mesons. By extrapolating into the $q \bar{q}$ QED sector in which a quark and an antiquark interact with the QED interaction, we find an open string isoscalar $I\left(J^{\pi}\right)=0\left(0^{-}\right)$QED meson state at $17.9 \pm 1.5 \mathrm{MeV}$ and an isovector $\left(I\left(J^{\pi}\right)=1\left(0^{-}\right), I_{3}=0\right)$ QED meson state at $36.4 \pm 3.8 \mathrm{MeV}$. The predicted masses of the isoscalar and isovector QED mesons are close to the masses of the hypothetical X17 and E38 particles observed recently, making them good candidates for these particles. The decay products of QED mesons may show up as excess $e^{+} e^{-}$and $\gamma \gamma$ pairs in the anomalous soft photon phenomenon associated with hadron productions in high-energy hadron-proton collisions and $e^{+}-e^{-}$annihilations. Measurements of the invariant masses of excess $e^{+} e^{-}$and $\gamma \gamma$ pairs will provide tests for the existence of the open string $q \bar{q}$ QED mesons. An assembly of gravitating QED mesons are expected to emit electron-positron pairs and/or gamma rays and their decay energies and lifetimes will be modified by their gravitational binding energies. Consequently, a self-gravitating isoscalar QED meson assembly whose mass $M$ and radius $R$ satisfy $\left(M / M_{\odot}\right) /\left(R / R_{\odot}\right) \gtrsim 4.71 \times 10^{5}$ will not produce electron-positron pairs nor gamma rays and may be a good candidate for the primordial dark matter.

KEYwords: Phenomenological Models, QCD Phenomenology

ARXIV EPRINT: 2001.04864 


\section{Contents}

1 Introduction 1

2 Open string QCD and QED states of $q \bar{q}$ systems 4

2.1 Quarks and antiquarks interacting with the QCD and QED interactions 4

2.2 Bosonization of QCD and QED for $q \bar{q}$ systems in $1+1$ dimensions 5

2.3 Orthogonal transformation to $q \bar{q}$ flavor eigenstates 9

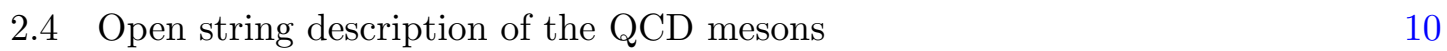

$\begin{array}{ll}2.5 & \text { Open string description of the QED mesons } \\ \end{array}$

3 Production of the QCD and QED mesons 16

4 Transverse momentum distribution of anomalous soft photons $\quad 18$

5 Behavior of a massive QED meson assembly 21

6 Conclusions and discussions $\quad 23$

\section{Introduction}

Recent observation [1] of a light, neutral boson decaying into an $e^{+} e^{-}$pair with a mass of about $17 \mathrm{MeV}$, in the decay of the $I\left(J^{\pi}\right)=0\left(1^{+}\right)$state of ${ }^{8} \mathrm{Be}$ at Atomki, has generated a great deal of interest [2-15]. Supporting evidence for this hypothetical X17 particle has been reported recently in the decay of the excited $I\left(J^{\pi}\right)=0\left(0^{-}\right)$state of ${ }^{4} \mathrm{He}$ [16]. Earlier observations of similar $e^{+} e^{-}$pairs with invariant masses between 3 to $20 \mathrm{MeV}$ in the collision of nuclei with emulsion detectors have been reported [17-22]. Different theoretical interpretations, astrophysical implications, and experimental searches have been presented [2-15]. However, a definitive description of the X17 particle has not yet emerged.

The observations of the $e^{+} e^{-}$pair with an invariant mass of about $17 \mathrm{MeV}[1,16]$ may appear perplexing, equally perplexing have been numerous observations of excess $e^{+} e^{-}$ pairs, labeled as "anomalous soft photons", whenever hadrons are produced in high-energy $K^{+} p[23,24], \pi^{+} p[24], \pi^{-} p$ [25, 26], $p p$ collisions [27], and $e^{+} e^{-}$annihilations [28-31]. Specifically in the DELPHI exclusive measurements in the decay of $Z^{0}$ in $e^{+} e^{-}$annihilations, the excess $e^{+} e^{-}$pairs have been observed to be proportionally produced when hadrons (mostly mesons) are produced [30,31], and they are not produced when hadrons are not produced in high-energy $e^{+}+e^{-} \rightarrow \mu^{+}+\mu^{-}$bremsstrahlung [29]. The transverse momenta of the excess $e^{+} e^{-}$pairs lie in the range of a few $\mathrm{MeV} / \mathrm{c}$ to many tens of $\mathrm{MeV} / \mathrm{c}$, corresponding to a mass scale of the anomalous soft photons in the range from a few $\mathrm{MeV}$ to many tens of $\mathrm{MeV}$. 
It happens as if the X17 particle and the anomalous soft photons are not perplexing enough, there occurs in addition the perplexing E38 boson particle with a mass of about $38 \mathrm{MeV}$ observed in the $\gamma \gamma$ invariant mass spectrum in high-energy $p \mathrm{C}, \mathrm{dC}, \mathrm{dCu}$ reactions at Dubna [32, 33]. The extra-ordinariness of these objects at the mass scale of many tens of $\mathrm{MeV}$ appears to place them outside the domain of the Standard Model. It is nonetheless important to explore here whether there may be a Standard Model description that can link these three perplexing objects in a coherent framework.

Many different models have been presented to describe the anomalous soft photons as arising from quantized bosons [34-36] or from a continuous spectrum [37-52]. We shall focus our attention on the quantized boson description of [34-36] which has the prospect of linking the anomalous soft photons with the X17 and E38 particles. We note that owing to the simultaneous and correlated production alongside with hadrons, a parent particle of the anomalous soft photons is likely to contain some elements of the hadron sector, such as a light quark and a light antiquark. ${ }^{1}$ The quark and antiquark carry color and electric charges and they interact mutually with the quantum chomodynamical (QCD) and quantum electrodynamical (QED) interactions. A parent particle of the anomalous soft photons cannot arise from the quark-antiquark pair interacting with the QCD interaction, because such an interaction will endow the pair with a mass much greater than the mass scale of the anomalous soft photons. We are left with the possibility of the quark and the antiquark interacting with the QED interaction. Such a possibility is further reinforced by the special nature of a confining gauge interaction, for which the greater the strength of the attractive confining interaction, the greater will be the mass of the composite particle it generates (see eq. (1.1) below), in contrast to a non-confining interaction in which the effect is just the opposite. Relative to the QCD interaction, the QED interaction will bring the quantized mass of a $q \bar{q}$ pair to the lower mass range of the anomalous soft photons. It was therefore proposed in [34] that a quark and an antiquark in a $q \bar{q}$ system interacting with the QED interaction may lead to new open string bound states (QED-meson states) with a mass of many tens of $\mathrm{MeV}$. These QED mesons may be produced simultaneously with the QCD mesons in the string fragmentation process in high-energy collisions [23-31], and the excess $e^{+} e^{-}$pairs may arise from the decays of these QED mesons. The predicted mass of the isoscalar $I\left(J^{\pi}\right)=0\left(0^{-}\right)$QED meson is close to the X17 mass of about $17 \mathrm{MeV}$. It is natural to inquire whether the hypothetical X17 particle may be the isoscalar $0\left(0^{-}\right)$ QED mesons predicted in [34]. It is also useful to inquire whether there can be additional experimental tests to confirm or refute such proposed QED mesons. For example, the mass of the isovector $\left(I\left(J^{\pi}\right)=0\left(0^{-}\right), I_{3}=0\right)$ QED meson predicted in [34] is close to the mass of the hypothetical E38 boson observed with an invariant mass of about $38 \mathrm{MeV}$ in highenergy $p \mathrm{C}, d \mathrm{C}, d \mathrm{Cu}$ reactions at Dubna $[32,33]$ and suggested earlier in $[53,54]$. There are furthermore possible $\gamma \gamma$ invariant mass structures at $10-15 \mathrm{MeV}$ and $38 \mathrm{MeV}$ in $p p$, and $\pi^{-} p$ reactions in COMPASS experiments [55-61]. Future investigations in the region of low $\gamma \gamma$ invariant masses will provide additional tests to confirm or refute the concept of the open string QED mesons.

\footnotetext{
${ }^{1}$ The elements of the hadron sector comprise of $u, d, c, s, b, t$ quarks, antiquarks, and gluons. The mass scale of the anomalous soft photons excludes all but the $u$ and $d$ quarks and antiquarks as possible constituents of the parent particles of the anomalous soft photons.
} 
It is instructive to re-examine the theoretical basis for the possible occurrence of such open string $q \bar{q}$ "QED mesons" as proposed in [34]. Because a quark $q$ and an antiquark $\bar{q}$ cannot be isolated, the intrinsic motion of a composite $q \bar{q}$ system in the lowest-energy states in $3+1$ dimensions lies predominantly in $1+1$ dimensions, as in an open string with the quark and the antiquark at its two ends. The approximate validity for the open string description for the lowest-energy $q \bar{q}$ systems is theoretically supported by the dual-resonance model [62], Nambu and Goto meson string model [63, 64], 'tHooft's two-dimensional meson model [65, 66], the classical yo-yo string model and the Lund model [67-70], the 2D insideoutside cascade model of Bjorken [71], Casher, Kogut, and Susskind [72], and lattice gauge theories [73-75]. The open string description of a flux tube in hadron production at high energies is experimentally supported by the limiting average transverse momentum and a rapidity plateau [71, 72, 76-80] in high-energy $e^{+}-e^{-}$annihilations [81-85] and $p p$ collisions [86]. To study approximately the lowest-energy bound states of $q \bar{q}$ systems with light quarks, it is reasonable to truncate the gauge field theories from $3+1$ dimensions to $1+1$ dimensions by idealizing the three-dimensional flux tube as a structureless one-dimensional open string, with the information on the structure of the flux tube stored into the coupling constant of the interaction in the lower $1+1$ dimensions. Whatever deviations from such a truncation can be considered as perturbations. The approximate validity of such a truncation will need to be tested by confronting its theoretical results with experiment.

In $1+1$ dimensions, Schwinger already showed that a massless fermion and an antifermion interacting with a gauge interaction give rise to a bound boson [87, 88]. If one identifies Schwinger's massless fermion and antifermion as a light quark and a light antiquark, one will reach the conclusion that a gauge interaction between the quark and the antiquark in $1+1$ dimensions leads to a confined and bound boson state with a mass $m$, related to the gauge field coupling constant $g_{2 \mathrm{D}}$ by $[87,88]$

$$
m^{2}=\frac{g_{2 \mathrm{D}}^{2}}{\pi}
$$

which shows that the mass $m$ of the bound boson increases as the strength $g_{2 \mathrm{D}}$ of the interaction increases, whether it be the QED or the QCD interaction. ${ }^{2}$

We need an important relationship to ensure that the boson mass calculated in the lower $1+1$ dimensions can appropriately represent the mass of a physical boson in $3+1$ dimensions. In the physical world of $3+1$ dimensions, the one-dimensional $q \bar{q}$ open string without a structure is in fact an idealization of a flux tube with a transverse radius $R_{T}$. The boson masses calculated in $1+1$ dimensions can represent physical boson masses, when the structure of the flux tube is properly taken into account. Upon considering the structure of the flux tube in the physical $3+1$ dimensions, we find that the coupling constant $g_{2 \mathrm{D}}$ in lower $1+1$ dimensions is related to the physical coupling constants $g_{4 \mathrm{D}}$ in $3+1$ dimensions by $[34,80,92]$

$$
\left(g_{2 \mathrm{D}}\right)^{2}=\frac{1}{\pi R_{T}^{2}}\left(g_{4 \mathrm{D}}\right)^{2}=\frac{4 \alpha_{4 \mathrm{D}}}{R_{T}^{2}},
$$

\footnotetext{
${ }^{2}$ For a pedagogical derivation of Schwinger's result of eq. (1.1), see for example, Chapter 6 of [79]. For recent generalizations and extensions of the Schwinger model, see [89-91].
} 
whose qualitative consistency can be checked by dimensional analysis. Thus, when the dynamics in the higher dimensional $3+1$ space-time is approximated as dynamics in the lower $1+1$ dimensions, information on the flux tube structure is stored in the multiplicative conversion factor $1 / \pi R_{T}^{2}$ in the above equation that relates the physical coupling constant square $\left(g_{4 D}\right)^{2}$ in $3+1$ dimensions to the new coupling constant square $\left(g_{2 D}\right)^{2}$ in $1+1$ dimensions. As a consequence, there is no loss of the relevant physical information. The boson mass $m$ determined in $1+1$ dimensions is the physical mass related to the physical coupling constant $\alpha_{4 \mathrm{D}}=\left(g_{4 \mathrm{D}}\right)^{2} / 4 \pi$ and the flux tube radius $R_{T}$ by

$$
m^{2}=\frac{4 \alpha_{4 \mathrm{D}}}{\pi R_{T}^{2}}
$$

With $\alpha_{4 \mathrm{D}}^{\mathrm{QED}}=\alpha_{\mathrm{QED}}=1 / 137, \alpha_{4 \mathrm{D}}^{\mathrm{QCD}}=\alpha_{s} \sim 0.6$ from hadron spectroscopy [93-96], and $R_{T} \sim 0.4 \mathrm{fm}$ from lattice QCD calculations [75] and $\left\langle p_{T}^{2}\right\rangle$ of produced hadrons in highenergy $e^{+} e^{-}$annihilations [83], we estimate the masses of the open string QCD and QCD mesons to be

$$
m^{\mathrm{QCD}} \sim 431 \mathrm{MeV}, \quad \text { and } m^{\mathrm{QED}} \sim 47 \mathrm{MeV} .
$$

The above mass scales provide an encouraging guide for the present task of a quantitative description of the QCD and QED mesons as open strings, using QCD and QED gauge field theories in $1+1$ dimensions. Of course, the approximate validity of such a truncation of the $q \bar{q}$ systems from $3+1$ dimensions to $1+1$ dimensions must be tested by direct confrontation with experimental data, as will be carried out in the next section.

\section{Open string QCD and QED states of $q \bar{q}$ systems}

\subsection{Quarks and antiquarks interacting with the QCD and QED interactions}

A quark and an antiquark carry color and electric charges. They interact with the QCD and the QED interactions, which are independent interactions with different gauge symmetries, commutation properties, and coupling constants. The QED interaction is a U(1) gauge interaction, whereas the QCD interaction is an $\mathrm{SU}(3)$ gauge interaction. They possess different generators and give rise to bound boson states of quarks and antiquarks at different state energies, as the order-of-magnitude estimates in (1.4) indicate.

We would like to review and extend our earlier work [34] on the $q \bar{q}$ bound states with QCD and QED interactions in a single framework. We wish to extend our considerations from two flavors to three flavors for the QCD interaction so that $\pi^{0}, \eta$, and $\eta^{\prime}$ can be adequately described. A successful description of these hadrons as open string $q \bar{q}$ QCD mesons will lend support for its theoretical extrapolation into the unknown sector of $q \bar{q}$ QED mesons.

Accordingly, we introduce an enlarged U(3) group that is the union of the color SU(3) QCD subgroup and the electromagnetic U(1) QED subgroup [34]. The generator $t^{0}$ for the $\mathrm{U}(1)$ subgroup is

$$
t^{0}=\frac{1}{\sqrt{6}}\left(\begin{array}{lll}
1 & 0 & 0 \\
0 & 1 & 0 \\
0 & 0 & 1
\end{array}\right)
$$


which adds on to the eight standard generators of the SU(3) subgroup, $\left\{t^{1}, \ldots t^{8}\right\}$, to form the nine generators of the $\mathrm{U}(3)$ group. They satisfy $2 \operatorname{tr}\left\{t^{a} t^{b}\right\}=\delta^{a b}$ for $a, b=0,1, \ldots, 8$. The two subgroups of $\mathrm{U}(3)$ differ in their coupling constants and communicative properties. We consider quarks with $N_{f}^{\lambda}$ number of flavors where $f=u, d, s=1,2,3$ is the flavor label, and the superscript $\lambda$ is the interaction label with $\lambda=0$ for QED and $\lambda=1$ for QCD. Because of the mass scale of (1.4), we have $m^{\mathrm{QCD}} \gg\left\{m_{u}, m_{d}, m_{s}\right\}$ and $m^{\mathrm{QED}} \gg\left\{m_{u}, m_{d}\right\}$, and we can choose $N_{f}^{\mathrm{QED}}=N_{f}^{0}=2$ and $N_{f}^{\mathrm{QCD}}=N_{f}^{1}=3$.

We start with $3+1$ dimensional space-time $x^{\mu}$, with $\mu=0,1,2,3$. The dynamical variables are the $\mathrm{U}(3)$ gauge fields $A_{\mu}=\sum_{a} A_{\mu}^{a} t^{a}$ and the quark fields $\psi_{f}^{i}$ where $i$ is the color index with $i=1,2,3$. We use the summation convention over repeated indices except when the summation symbols are needed to avoid ambiguities. For brevity of notations, the indices $\{a, f\}$ and the superscript interaction labels $\{\lambda, Q C D, Q E D\}$ in various quantities are often implicitly understood except when they are needed to resolve ambiguities. The coupling constants $g_{f}^{a}$ are given explicitly by

$$
\begin{aligned}
g_{u}^{0} & =-Q_{u} g_{4 \mathrm{D}}^{\mathrm{QED}}, \quad g_{d}^{0}=-Q_{d} g_{4 \mathrm{D}}^{\mathrm{QED}} & & \text { for } \mathrm{QED}, \\
g_{\{u, d, s\}}^{\{1, \ldots, 8\}} & =Q_{\{u, d, s\}}^{\mathrm{QCD}} g_{4 \mathrm{D}}^{\mathrm{QCD}} & & \text { for QCD }
\end{aligned}
$$

where we have introduced the charge numbers $Q_{u}^{\mathrm{QED}}=2 / 3, Q_{d}^{\mathrm{QED}}=-1 / 3, Q_{u}^{\mathrm{QCD}}=Q_{d}^{\mathrm{QCD}}=Q_{s}^{\mathrm{QCD}}=1$. The Lagrangian density for the system is

$$
\begin{aligned}
\mathcal{L} & =\bar{\psi}(i \not D) \psi-\frac{1}{4} F_{\mu \nu} F^{\mu \nu}-m \bar{\psi} \psi, \\
\text { where } \quad i \not D & =\gamma^{\mu}\left(i \not \partial+g A_{\mu}\right), \\
F_{\mu \nu} & =\partial_{\mu} A_{\nu}-\partial_{\nu} A_{\mu}-i g\left[A_{\mu}, A_{\nu}\right], \quad F_{\mu \nu}=F_{\mu \nu}^{a} t^{a} .
\end{aligned}
$$

The equation of motion for the gauge field $A_{\mu}$ is

$$
D_{\mu} F^{\mu \nu}=\partial_{\mu} F^{\mu \nu}-i g\left[A_{\mu}, F^{\mu \nu}\right]=g j^{\nu}, \quad j^{\nu}=j^{\nu a} t^{a}, \quad j^{\nu a}=2 \operatorname{tr} \bar{\psi}_{f} \gamma^{\nu} t^{a} \psi_{f} .
$$

As a quark and an antiquark cannot be isolated, the intrinsic motion of the quark and the antiquark in the lowest-energy $q \bar{q}$ systems in $3+1$ dimensions lies predominantly in $1+1$ dimensions, as discussed in string models of mesons [34, 35, 62-72, 80, 92, 97, 98]. We shall therefore approximate the gauge field theory in $3+1$ dimensions by the gauge field theory in $1+1$ dimensions where the coupling constant $g$ will be implicitly taken to be $g_{2 \mathrm{D}}$. It is necessary to keep in mind that the information on the structure of flux tube radius $R_{T}$ is stored in the multiplicative factor $1 /\left(\sqrt{\pi} R_{T}\right)$ that converts the physical coupling constant $g_{4 \mathrm{D}}$ to the new coupling $g_{2 \mathrm{D}}$ in the lower dimensions as given by (1.2).

\subsection{Bosonization of QCD and QED for $q \bar{q}$ systems in $1+1$ dimensions}

We wish to search for bound states arising from the interaction of the color and electric charges of the quarks and antiquarks in QCD and QED in the strong coupling limit in $1+1$ dimensions. The bound states can be searched by the method of bosonization in which 
the stability of the boson states can be examined by the values of the square of the boson mass, with residual sine-Gordon interactions that depend on the quark mass [98-109].

The $\mathrm{U}(3)$ gauge interactions under consideration contain the non-Abelian color $\mathrm{SU}(3)$ interactions. Consequently the bosonization of the color degrees of freedom should be carried out according to the method of non-Abelian bosonization which preserves the gauge group symmetry [100].

While we use non-Abelian bosonization for the U(3) gauge interactions, we shall follow Coleman to treat the flavor degrees of freedom as independent degrees of freedom [98, 102, 103, 108, 109]. This involves keeping the flavor labels in the bosonization without using the flavor group symmetry. Although such bosonization in the flavor sector obscures the isospin and other flavor symmetry in QCD, the QCD isospin and other flavor symmetry are still present. They can be recovered by complicated non-linear general transformations [98, 99], or by using explicit physical $q \bar{q}$ multi-flavor eigenstates as a linear combination of flavor components. As such a bosonization method is simple only for neutral $q \bar{q}$ systems with isospin component $I_{3}=0$, we shall limit our attention to such systems. We shall study only $q \bar{q}$ systems with total spin $S=0$.

As in any method of bosonization, the non-Abelian method will succeed for systems that contain stable and bound boson states with relatively weak residual interactions. Thus, not all the degrees of freedom available to the bosonization technique will lead to good boson states with these desirable properties. For example, some of the bosonization degrees of freedom in color $\mathrm{SU}(3)$ may correspond to bosonic excitations into colored objects of two-fermion complexes and may not give rise to stable bosons. It is important to judiciously search for those boson degrees of freedom that will eventually lead to stable and bound bosons. Keeping this perspective in our mind, we can examine the non-Abelian bosonization of the system under the $\mathrm{U}(3)$ interactions. The non-Abelian bosonization program consists of introducing boson fields $\phi^{a}$ to describe an element $u$ of the U(3) group and showing subsequently that these boson fields lead to stable bosons with finite or zero masses.

In the non-Abelian bosonization, the current $j_{ \pm}$in the light-cone coordinates, $x^{ \pm}=$ $\left(x^{0} \pm x^{3}\right) / \sqrt{2}$, is bosonized as [100]

$$
\begin{aligned}
& j_{+}=(i / 2 \pi) u^{-1}\left(\partial_{+} u\right), \\
& j_{-}=-(i / 2 \pi)\left(\partial_{-} u\right) u^{-1} .
\end{aligned}
$$

An element of the U(1) subgroup of the $\mathrm{U}(3)$ group can be represented by the boson field $\phi^{0}$

$$
u=\exp \left\{i 2 \sqrt{\pi} \phi^{0} t^{0}\right\} .
$$

Such a bosonization poses no problem as it is an Abelian subgroup. It will lead to a stable boson as in Schwinger's QED2.

To carry out the bosonization of the color SU(3) subgroup, we need to introduce boson fields to describe an element $u$ of SU(3). There are eight $t^{a}$ generators which provide eight degrees of freedom. We may naively think that for the non-Abelian bosonization of SU(3), 
we should introduce eight boson fields $\phi^{a}$ to describe $u$ by

$$
u=\exp \left\{i 2 \sqrt{\pi} \sum_{a=1}^{8} \phi^{a} t^{a}\right\} .
$$

However, a general variation of the element $\delta u / \delta x^{ \pm}$will lead to quantities that in general do not commute with $u$ and $u^{-1}$, resulting in $j_{ \pm}$currents in eqs. (2.5) that are complicated non-linear admixtures of the boson fields $\phi^{a}$. It will be difficult to look for stable boson states with these currents.

We can guide ourselves to a situation that has a greater chance of finding stable bosons by examining the bosonization problem from a different viewpoint. We can pick a unit generator $\tau^{1}=\sum_{a=1}^{8} n_{a} t^{a}$ with $n_{a}=2 \operatorname{tr}\left(\tau^{1} t^{a}\right)$ oriented in any direction of the eight-dimensional generator space and we can describe an $\mathrm{SU}(3)$ group element $u$ by an amplitude $\phi^{1}$ and the unit vector $\tau^{1}$,

$$
u=\exp \left\{i 2 \sqrt{\pi} \phi^{1} \tau^{1}\right\}
$$

The boson field $\phi^{1}$ describes one degree of freedom, and the direction cosines $\left\{n^{a}, a=\right.$ $1, \ldots, 8\}$ of the unit vector $\tau^{1}$ describe the other seven degrees of freedom. A variation of the amplitude $\phi^{1}$ in $u$ while keeping the unit vector orientation fixed will lead to a variation of $\delta u / \delta x^{ \pm}$that will commute with $u$ and $u^{-1}$ in the bosonization formula (2.5), as in the case with an Abelian group element. It will lead to simple currents and stable QCD bosons with well defined masses, which will need to be consistent with experimental QCD meson data. On the other hand, a variation of $\delta u / \delta x^{ \pm}$in any of the other seven orientation angles of the unit vector $\tau^{1}$ will lead to $\delta u / \delta x^{ \pm}$quantities along other $t^{a}$ directions with $a=\{1, \ldots, 8\}$. These variations of $\delta u / \delta x^{ \pm}$will not in general commute with $u$ or $u^{-1}$. They will lead to $j_{ \pm}$currents that are complicated non-linear functions of the eight degrees of freedom. We are therefore well advised to search for stable bosons by varying only the amplitude of the $\phi^{1}$ field, keeping the orientation of the unit vector fixed, and forgoing the other seven orientation degrees of freedom. For the U(3) group, there is in addition the group element $u=\exp \left\{i 2 \sqrt{\pi} \phi^{0} t^{0}\right\}$ from the QED U(1) subgroup. Combining both U(1) and $\mathrm{SU}(3)$ subgroups, we can represent an element $u$ of the $\mathrm{U}(3)$ group by $\phi^{0}$ from QED and $\phi^{1}$ from QCD as [34]

$$
u=\exp \left\{i 2 \sqrt{\pi} \phi^{0} \tau^{0}+i 2 \sqrt{\pi} \phi^{1} \tau^{1}\right\}
$$

where we have re-labeled $t^{0}$ as $\tau^{0}$. The superscripts $\lambda=\{0,1\}$ on the right hand side of the above equation is the interaction label with $\lambda=\{0,1\}$ for QED and QCD, respectively, and $2 \operatorname{tr}\left(\tau^{\lambda} \tau^{\lambda^{\prime}}\right)=\delta^{\lambda \lambda^{\prime}}$. When we write out the flavor index explicitly, we have

$$
u_{f}=\exp \left\{i 2 \sqrt{\pi} \phi_{f}^{0} \tau^{0}+i 2 \sqrt{\pi} \phi_{f}^{1} \tau^{1}\right\} .
$$

From (2.5a) and (2.5b), we obtain

$$
\begin{aligned}
j_{f \pm} & =\mp \frac{1}{\sqrt{\pi}}\left[\left(\partial_{ \pm} \phi_{f}^{0}\right) \tau^{0}+\left(\partial_{ \pm} \phi_{f}^{1}\right) \tau^{1}\right] \quad \text { when all } Q_{f}^{\lambda}=1, \\
& =\mp \frac{1}{\sqrt{\pi}}\left[Q_{f}^{0}\left(\partial_{ \pm} \phi_{f}^{0}\right) \tau^{0}+Q_{f}^{1}\left(\partial_{ \pm} \phi_{f}^{1}\right) \tau^{1}\right] \text { when we include charge number } Q_{f}^{\lambda} .
\end{aligned}
$$


The Maxwell equation in light-cone coordinates is

$$
\partial_{\mu} \partial^{\mu} A^{ \pm}-\partial_{ \pm} \partial_{\mu} A^{\mu}=g j^{ \pm}
$$

We shall use the Lorenz gauge

$$
\partial_{\mu} A^{\mu}=0
$$

then the solution of the gauge field is

$$
A^{ \pm}=\frac{g}{2 \partial_{+} \partial_{-}} j^{ \pm}
$$

Interaction energy $H_{\text {int }}$ is

$$
\begin{aligned}
H_{\text {int }} & =\frac{g}{2} \int d x^{+} d x^{-} 2 \operatorname{tr}(j \cdot A)=\frac{g}{2} \int d x^{+} d x^{-} 2 \operatorname{tr}\left(j^{+} A^{-}+j^{-} A^{+}\right) \\
& =\frac{g}{2} \int d x^{+} d x^{-} 2 \operatorname{tr}\left(j^{+} \frac{g}{2 \partial_{+} \partial_{-}} j^{-}+j^{-} \frac{g}{2 \partial_{+} \partial_{-}} j^{+}\right) .
\end{aligned}
$$

We integrate by parts, include the charge numbers and the interaction dependency of the coupling constant, $g_{2 \mathrm{D}}^{\lambda}=g^{\lambda}$, and we obtain the contribution to the Hamiltonian density from the confining interaction between the constituents, $H_{\text {int }}=\int d x^{+} d x^{-} \mathcal{H}_{\text {int }}\left(\phi_{f}^{\lambda}\right)$,

$$
\mathcal{H}_{\text {int }}\left(\phi_{f}^{\lambda}\right)=\frac{1}{2}\left[\frac{\left(g_{2 \mathrm{D}}^{0}\right)^{2}}{\pi}\left(\sum_{f}^{N_{f}} Q_{f}^{0} \phi_{f}^{0}\right)^{2}+\frac{\left(g_{2 \mathrm{D}}^{1}\right)^{2}}{\pi}\left(\sum_{f}^{N_{f}} Q_{f}^{1} \phi_{f}^{1}\right)^{2}\right],
$$

which matches the results of $[98,108]$. For the mass bi-linear term, we follow Coleman [98] and Witten [100] and bosonized it as

$$
\begin{aligned}
m_{f}: \bar{\psi}_{f} \psi_{f}: & \rightarrow\left(-\frac{e^{\gamma}}{2 \pi}\right) \mu m_{f} 2 \operatorname{tr}\left(\frac{u_{f}+u_{f}^{-1}}{2}\right) \\
& =\left(-\frac{e^{\gamma}}{2 \pi}\right) \mu m_{f} 2 \operatorname{tr} \cos \left(2 \sqrt{\pi} \phi_{f}^{0} \tau^{0}+2 \sqrt{\pi} \phi_{f}^{1} \tau^{1}\right)
\end{aligned}
$$

where $\gamma=0.5772$ is the Euler constant, and $\mu$ is an unknown mass scale that arises from the bosonization of the scalar density $\bar{\psi} \psi$ and is solution-dependent [98]. When we sum over flavors, we get the contribution to the Hamiltonian density from quark masses,

$$
H_{\mathrm{m}}=\int d x^{+} d x^{-} \mathcal{H}_{\mathrm{m}}\left(\phi_{f}^{\lambda}\right)
$$

where

$$
\begin{aligned}
\mathcal{H}_{\mathrm{m}}\left(\phi_{f}^{\lambda}\right) & =e^{\gamma} \mu \sum_{f} m_{f}\left[\left(\phi_{f}^{0}\right)^{2}+\left(\phi_{f}^{1}\right)^{2}+\ldots\right] \quad \text { when } \mu \text { is independent of interaction, } \\
& =e^{\gamma} \sum_{f} m_{f}\left[\mu^{0}\left(\phi_{f}^{0}\right)^{2}+\mu^{1}\left(\phi_{f}^{1}\right)^{2}+\ldots\right] \text { when } \mu \text { depends on interaction. }
\end{aligned}
$$


Finally, for the kinematic term, we bosonize it as [100, 101]

$$
\left.: \bar{\psi} i \not \partial \psi: \quad \rightarrow \quad \frac{1}{8 \pi}\left\{2 \operatorname{tr}\left[\partial_{\mu} u\right)\left(\partial^{\mu} u^{-1}\right)\right]\right\}+n \Gamma
$$

where $n \Gamma$ is the Wess-Zumino term which vanishes for $u$ of (2.9) containing commuting elements $\tau^{0}$ and $\tau^{1}$. We get the kinematic contribution

$$
\left.H_{\text {kin }}=\int d x^{0} d x^{1} \mathcal{H}_{\text {kin }}=\int d x^{0} d x^{1} \sum_{f} \frac{1}{8 \pi}\left\{2 \operatorname{tr}\left[\partial_{\mu} u_{f}\right)\left(\partial^{\mu} u_{f}^{-1}\right)\right]\right\}
$$

where

$$
\mathcal{H}_{\text {kin }}\left(\phi_{f}^{\lambda}\right)=\frac{1}{2} \sum_{f}\left[\partial_{\mu} \phi_{f}^{0} \partial^{\mu} \phi_{f}^{0}+\partial_{\mu} \phi_{f}^{1} \partial^{\mu} \phi_{f}^{1}\right]=\frac{1}{2} \sum_{\lambda} \sum_{f}\left[\left(\Pi_{f}^{\lambda}\right)^{2}+\left(\partial_{x} \phi_{f}^{\lambda}\right)^{2}\right],
$$

and $\Pi_{f}^{\lambda}$ is the momentum conjugate to $\phi_{f}^{\lambda}$. The total Hamiltonian density in terms of $\phi_{f}^{\lambda}$ is

$$
\mathcal{H}\left(\phi_{f}^{\lambda}\right)=\mathcal{H}_{\text {kin }}\left(\phi_{f}^{\lambda}\right)+\mathcal{H}_{\text {int }}\left(\phi_{f}^{\lambda}\right)+\mathcal{H}_{\mathrm{m}}\left(\phi_{f}^{\lambda}\right),
$$

where $\mathcal{H}_{\text {kin }}\left(\phi_{f}^{\lambda}\right), \mathcal{H}_{\text {int }}\left(\phi_{f}^{\lambda}\right), \mathcal{H}_{\mathrm{m}}\left(\phi_{f}^{\lambda}\right)$ are given by eqs. (2.22), (2.16), and (2.19b) respectively.

\subsection{Orthogonal transformation to $q \bar{q}$ flavor eigenstates}

We consider $q \bar{q}$ systems with dynamical flavor symmetry that lead to flavor eigenstates as a linear combination of states with different flavor amplitudes. Such eigenstates arise from additional considerations of isospin invariance, SU(3) flavor symmetry, and configuration mixing. As a result of such considerations in flavor symmetry and configuration mixing, the physical eigenstates $\Phi_{i}$ can be quite generally related to various flavor components $\phi_{f}$ by a linear orthogonal transformation as

$$
\Phi_{i}^{\lambda}=\sum_{f} D_{i f}^{\lambda} \phi_{f}^{\lambda}
$$

The orthogonal transformation matrix $D_{i f}^{\lambda}$ obeys $\left(D^{\lambda}\right)^{-1}=\left(D^{\lambda}\right)^{\dagger}$ with $\left(\left(D^{\lambda}\right)^{\dagger}\right)_{f i}=D_{i f}^{\lambda}$. The inverse transformation is

$$
\phi_{f}^{\lambda}=\sum_{i} D_{i f}^{\lambda} \Phi_{i}^{\lambda}
$$

Upon substituting the above equation into (2.23), we obtain the total Hamiltonian density in terms of the physical flavor state $\Phi_{i}^{\lambda}$ as

$$
\mathcal{H}\left(\Phi_{f}^{\lambda}\right)=\left[\mathcal{H}_{\text {kin }}\left(\Phi_{i}^{\lambda}\right)+\mathcal{H}_{\text {int }}\left(\Phi_{i}^{\lambda}\right)+\mathcal{H}_{\mathrm{m}}\left(\Phi_{i}^{\lambda}\right)\right],
$$

where

$$
\begin{aligned}
\mathcal{H}_{\text {kin }}\left(\Phi_{i}^{\lambda}\right) & =\frac{1}{2} \sum_{\lambda} \sum_{i}\left[\partial_{\mu} \Phi_{i}^{\lambda} \partial^{\mu} \Phi_{i}^{\lambda}\right]=\frac{1}{2} \sum_{\lambda} \sum_{i}\left[\left(\Pi_{i}^{\lambda}\right)^{2}+\left(\partial_{x} \Phi_{i}^{\lambda}\right)^{2}\right], \\
\mathcal{H}_{\text {int }}\left(\Phi_{i}^{\lambda}\right) & =\frac{1}{2}\left[\sum_{\lambda} \frac{\left(g_{2 \mathrm{D}}^{\lambda}\right)^{2}}{\pi}\left(\sum_{f} Q_{f}^{\lambda} \sum_{i} D_{i f}^{\lambda} \Phi_{i}^{\lambda}\right)^{2}\right], \\
\mathcal{H}_{\mathrm{m}}\left(\Phi_{i}^{\lambda}\right) & =e^{\gamma} \sum_{f} m_{f}\left[\sum_{\lambda} \mu^{\lambda}\left(\sum_{i} D_{i f}^{\lambda} \Phi_{i}^{\lambda}\right)^{2}\right],
\end{aligned}
$$


where $\Pi_{i}^{\lambda}$ is the momentum conjugate to $\Phi_{i}^{\lambda}$. We can get the boson mass $m_{i}^{\lambda}$ of the physical state $\Phi_{i}^{\lambda}$ by expanding the potential energy term, $\mathcal{H}_{\text {int }}\left(\Phi_{i}^{\lambda}\right)+\mathcal{H}_{\mathrm{m}}\left(\Phi_{i}^{\lambda}\right)$, about the potential minimum located at $\Phi_{i}^{\lambda}=0$, up to the second power in $\left(\Phi_{i}^{\lambda}\right)^{2}$, as

$$
\mathcal{H}\left(\Phi_{i}^{\lambda}\right)=\sum_{\lambda} \sum_{i}\left[\frac{1}{2}\left(\Pi_{i}^{\lambda}\right)^{2}+\frac{1}{2}\left(\partial_{x} \Phi_{i}^{\lambda}\right)^{2}+\frac{1}{2}\left(m_{i}^{\lambda}\right)^{2}\left(\Phi_{i}^{\lambda}\right)^{2}\right]+\ldots,
$$

where

$$
\left(m_{i}^{\lambda}\right)^{2}=\left[\frac{\partial^{2}}{\partial\left(\Phi_{i}^{\lambda}\right)^{2}}\left[\mathcal{H}_{\mathrm{int}}\left(\left\{\Phi_{i}^{\lambda}\right\}\right)+\mathcal{H}_{\mathrm{m}}\left(\left\{\Phi_{i}^{\lambda}\right\}\right)\right]\right]_{\Phi_{0}^{\lambda}, \Phi_{1}^{\lambda}=0} .
$$

From eqs. (2.27b) and (2.27c), we find the squared mass $\left(m_{i}^{\lambda}\right)^{2}$ for the state $\Phi_{i}^{\lambda}$ of interaction $\lambda$ to be

$$
\left(m_{i}^{\lambda}\right)^{2}=\frac{\left(g_{2 \mathrm{D}}^{\lambda}\right)^{2}}{\pi}\left[\sum_{f}^{N_{f}} Q_{f}^{\lambda} D_{i f}^{\lambda}\right]^{2}+e^{\gamma} \sum_{f}^{N_{f}} m_{f} \mu^{\lambda}\left(D_{i f}^{\lambda}\right)^{2} .
$$

This mass formula includes the mixing of the configurations, and is applicable to QCD with three flavors. It is an improved and more general extension of the earlier mass formula in [34]. It should be reminded that the coupling constant $g_{2 \mathrm{D}}^{\lambda}$ in $1+1$ dimensions above is related to the coupling constant $g_{4 \mathrm{D}}^{\lambda}$ in $3+1$ dimensions by the flux tube radius in (1.2). A positive definite value of $\left(m_{i}^{\lambda}\right)^{2}$, which is ensured by the positive quantities on the right hand side of (2.30), indicates that the boson from such an interacting system of $q$ and $\bar{q}$ are stable bosons.

The two terms on the right hand side of (2.30) receive contributions from different physical sources. The first term, the "massless quark limit" or alternatively the "confining interaction term", arises from the confining interaction between the quark and the antiquark. The second term arises from quark masses and the quark condensate, $\left\langle\sum_{f} \bar{\psi}_{f} \psi_{f}\right\rangle$. It can be called the "quark mass term" or the "quark condensate term". If one labels the square root of the first term in (2.30) as the confining interaction mass and the square root of the second term as the condensate mass, then the hadron mass obeys a Pythagoras theorem with the hadron mass as the hypotenuse and the confining interaction mass and the condensate mass as two sides of a right triangle.

\subsection{Open string description of the QCD mesons}

QCD has an approximate $\mathrm{SU}(3)_{L} \times \mathrm{SU}(3)_{R}$ chiral symmetry and also an approximate flavor $\mathrm{U}(3) \times \mathrm{U}(3)$ symmetry. If the axial symmetry is realized as the Goldstone mode as a result of the spontaneous chiral symmetry breaking, then one would naively expect the singlet isoscalar $\eta^{\prime}$ particle to have a mass comparable to the pion mass. Experimentally, there is the $\mathrm{U}_{A}(1)$ anomaly [110-114] in which the $\eta^{\prime}$ mass of $957.8 \mathrm{MeV}$ is so much higher than the $\pi$ mass. On the basis of the Schwinger model, Kogut, Susskind, and Sinclair [110112 ] suggested that such a $\mathrm{U}_{A}(1)$ anomaly arises from the long-range confinement between the quark and the antiquark, as the $\eta^{\prime}$ acquires a large mass from the long-range confining interaction between a quark and an antiquark. The long range gauge interaction affects not 
only $\eta^{\prime}$ mass but also the other pseudoscalar $\pi^{0}$, and $\eta$ masses, and there are furthermore the effects of quark rest masses, and the configuration mixing between $\eta$ and $\eta^{\prime}$. We would like to show that when these effects are properly taken into account, the pseudoscalar particles $\pi^{0}, \eta$, and $\eta^{\prime}$ can indeed be adequately described as open string QCD mesons.

Accordingly, in this subsection (2.4) to study QCD mesons, we restrict ourselves to the QCD interaction with the interaction label superscript set implicitly to $\lambda=1$ for QCD. Equation (1.4) indicates that the mass scale $m^{\mathrm{QCD}} \sim 431 \mathrm{MeV} \gg m_{u}, m_{d}, m_{s}$. It is necessary to include $u, d$, and $s$ quarks with $N_{f}=3$ in the analysis of open strings QCD mesons.

We denote $\phi_{1}=|u \bar{u}\rangle, \phi_{2}=|d \bar{d}\rangle$, and $\phi_{3}=|s \bar{s}\rangle$, and assume the standard quark model description of $\left|\pi^{0}\right\rangle,|\eta\rangle$, and $\left|\eta^{\prime}\right\rangle$ in terms of flavor octet and flavor singlet states, with the mixing of the $|\eta\rangle$ and $\left|\eta^{\prime}\right\rangle$ represented by a mixing angle $\theta_{P}$ [117]. The physical states of $\left|\pi^{0}\right\rangle,|\eta\rangle$, and $\left|\eta^{\prime}\right\rangle$ can be represented in terms of the flavor states $\phi_{1}, \phi_{2}$ and $\phi_{3}$ by

$$
\begin{aligned}
& \left|\pi^{0}\right\rangle=\Phi_{1}=\frac{\phi_{1}-\phi_{2}}{\sqrt{2}} \\
& |\eta\rangle=\Phi_{2}=\left|\eta_{8}\right\rangle \cos \theta_{P}-\left|\eta_{0}\right\rangle \sin \theta_{P} \\
& \left|\eta^{\prime}\right\rangle=\Phi_{3}=\left|\eta_{8}\right\rangle \sin \theta_{P}+\left|\eta_{0}\right\rangle \cos \theta_{P}
\end{aligned}
$$

where

$$
\begin{aligned}
\left|\eta_{8}\right\rangle & =\frac{\phi_{1}+\phi_{2}-2 \phi_{3}}{\sqrt{6}}, \\
\left|\eta_{0}\right\rangle & =\frac{\sqrt{2}\left(\phi_{1}+\phi_{2}+\phi_{3}\right)}{\sqrt{6}} .
\end{aligned}
$$

The physical states $\Phi_{i}=\sum_{f} D_{i f} \phi_{f}$ and the flavor component states $\phi_{f}$, are then related by

$$
\left(\begin{array}{c}
\Phi_{1} \\
\Phi_{2} \\
\Phi_{3}
\end{array}\right)=\left(\begin{array}{ccc}
\frac{1}{\sqrt{2}} & -\frac{1}{\sqrt{2}} & 0 \\
\frac{1}{\sqrt{6}}\left\{\cos \theta_{P}-\sqrt{2} \sin \theta_{P}\right\} & \frac{1}{\sqrt{6}}\left\{\cos \theta_{P}-\sqrt{2} \sin \theta_{P}\right\} & \frac{1}{\sqrt{6}}\left\{-2 \cos \theta_{P}-\sqrt{2} \sin \theta_{P}\right\} \\
\frac{1}{\sqrt{6}}\left\{\sin \theta_{P}+\sqrt{2} \cos \theta_{P}\right\} & \frac{1}{\sqrt{6}}\left\{\sin \theta_{P}+\sqrt{2} \cos \theta_{P}\right\} & \frac{1}{\sqrt{6}}\left\{-2 \sin \theta_{P}+\sqrt{2} \cos \theta_{P}\right\}
\end{array}\right)\left(\begin{array}{l}
\phi_{1} \\
\phi_{2} \\
\phi_{3}
\end{array}\right),
$$

with the inverse relation $\phi_{f}=\sum_{i=1}^{3} D_{i f} \Phi_{i}$,

$$
\left(\begin{array}{l}
\phi_{1} \\
\phi_{2} \\
\phi_{3}
\end{array}\right)=\left(\begin{array}{ccc}
\frac{1}{\sqrt{2}} & \frac{1}{\sqrt{6}}\left\{\cos \theta_{P}-\sqrt{2} \sin \theta_{P}\right\} & \frac{1}{\sqrt{6}}\left\{\sin \theta_{P}+\sqrt{2} \cos \theta_{P}\right\} \\
-\frac{1}{\sqrt{2}} & \frac{1}{\sqrt{6}}\left\{\cos \theta_{P}-\sqrt{2} \sin \theta_{P}\right\} & \frac{1}{\sqrt{6}}\left\{\sin \theta_{P}+\sqrt{2} \cos \theta_{P}\right\} \\
0 & \frac{1}{\sqrt{6}}\left\{-2 \cos \theta_{P}-\sqrt{2} \sin \theta_{P}\right\} & \frac{1}{\sqrt{6}}\left\{-2 \sin \theta_{P}+\sqrt{2} \cos \theta_{P}\right\}
\end{array}\right)\left(\begin{array}{l}
\Phi_{1} \\
\Phi_{2} \\
\Phi_{3}
\end{array}\right) .
$$

With color charge $Q_{\{u, s, d\}}^{\mathrm{QCD}}=1$, the mass formula (2.30) gives

$$
m_{i}^{2}=\left(\sum_{f=1}^{N_{f}} D_{i f}\right)^{2} \frac{4 \alpha_{s}}{\pi R_{T}^{2}}+\sum_{f=1}^{N_{f}} m_{f}\left(D_{i f}\right)^{2} e^{\gamma} \mu^{\mathrm{QCD}},
$$

yielding an effective color charge $Q_{i, \mathrm{eff}}=\left|\sum_{f=1}^{3}\left(D_{i f}\right)\right|$. 
For the pion state $\Phi_{1}$, we have $Q_{1 \text {,eff }}=\left|\sum_{f=1}^{3}\left(D_{1 f}\right)\right|=|1 / \sqrt{2}-1 / \sqrt{2}|=0$. The first term of the "massless quark limit" in the mass formula (2.33) gives a zero pion mass. When the quark masses are taken into account, we have $\left(D_{i f}\right)^{2}=1 / 2$. The only contribution comes from the second "quark condensate" term in (2.33). The mass formula (2.33) for the pion then gives

$$
m_{\pi}^{2}=\left(m_{u}+m_{d}\right) \frac{e^{\gamma} \mu^{\mathrm{QCD}}}{2},
$$

which is in the same form as the Gell-Mann-Oakes-Renner relation [115],

$$
m_{\pi}^{2}=\left(m_{u}+m_{d}\right) \frac{|\langle 0|\bar{q} q| 0\rangle|}{F_{\pi}^{2}}
$$

where $|\langle 0|\bar{q} q| 0\rangle|$ is the light $u$ and $d$ quark-antiquark condensate and $F_{\pi}$ is the pion decay constant [116]. Consequently, we can infer that the unknown mass scale $\mu^{\mathrm{QCD}}$ in the bosonization formula for QCD has indeed the physical meaning of the quark condensate. We can therefore identify $\mu^{\mathrm{QCD}}$ in the bosonization mass formula (2.33) for QCD as

$$
\frac{e^{\gamma} \mu^{\mathrm{QCD}}}{2}=\frac{|\langle 0|\bar{q} q| 0\rangle|}{F_{\pi}^{2}} .
$$

By such an identification and calibrating the pion mass to be the experimental mass $m_{\pi}$, the mass formula (2.33) for the pseudoscalar QCD mesons can be re-written as

$$
m_{i}^{2}=\left(\sum_{f=1}^{N_{f}} D_{i f}\right)^{2} \frac{4 \alpha_{s}}{\pi R_{T}^{2}}+m_{\pi}^{2} \sum_{f=1}^{N_{f}} \frac{m_{f}}{m_{u d}}\left(D_{i f}\right)^{2},
$$

where $m_{u d}=\left(m_{u}+m_{d}\right) / 2$. We are ready to test whether the $I_{3}=0, S=0$ hadrons of $\pi^{0}, \eta$ and $\eta^{\prime}$ can be appropriately described as open strings in the $1+1$ dimensional bosonization model. For these QCD mesons, there is a wealth of information on the matrix $D_{i f}$ that describes the composition of the physical states in terms of the flavor components, as represented by the mixing angle $\theta_{P}$ between the flavor octet and flavor singlet components of the $\mathrm{SU}(3)$ wave functions in $\eta$ and $\eta^{\prime}$ in (2.31b) and (2.31c). The ratio of the strange quark mass to the light $u$ and $d$ quark masses that is needed in the above mass formula is also known. From the tabulation in PDG [117], we find $\theta_{P}=-24.5^{\circ}$ and $m_{s} / m_{u d}=27.3_{-1.3}^{+0.7}$. The only free parameters left in the mass formula (2.37) are the strong interaction coupling constant $\alpha_{s}$ and the flux tube radius $R_{T}$.

For the value of $\alpha_{s}$, previous works on the non-perturabtive potential models use a value of $\alpha_{s}$ of the order of $0.4-0.6$ in hadron spectroscopy studies [93-96]. However, these potential models contain a linear confining interaction, in addition to the one-gluon exchange interaction involving $\alpha_{s}$. In contrast, the present simplified $1+1$ dimensional treatment uses only a single attractive gauge interaction, involving $\alpha_{s}$ and playing dual roles. We should be prepared to allow for a larger value of the strong coupling constant $\alpha_{s}$ in our case. We find that $\alpha_{s}=0.68$ gives a reasonable description of the masses of the mesons considered, and we can take the difference between this $\alpha_{s}$ value and the $\alpha_{s}$ value 


\begin{tabular}{|c|c|c|c|c|c|c|c|}
\hline & & $\mathrm{I}$ & $\mathrm{S}$ & {$\left[I\left(J^{\pi}\right)\right]$} & $\begin{array}{c}\text { Experimental } \\
\text { mass } \\
(\mathrm{MeV})\end{array}$ & $\begin{array}{c}\text { Semi-empirical } \\
\text { mass } \\
\text { formula } \\
(\mathrm{MeV})\end{array}$ & $\begin{array}{l}\text { Meson mass } \\
\text { in massless } \\
\text { quark limit } \\
\quad(\mathrm{MeV})\end{array}$ \\
\hline \multirow{3}{*}{$\begin{array}{l}\text { QCD } \\
\text { meson }\end{array}$} & $\pi^{0}$ & 1 & 0 & {$\left[1\left(0^{-}\right)\right]$} & $134.9768 \pm 0.0005$ & $134.9^{(a)}$ & 0 \\
\hline & $\eta$ & 0 & 0 & {$\left[0\left(0^{-}\right)\right]$} & $547.862 \pm 0.017$ & $498.4 \pm 39.8$ & $329.7 \pm 57.5$ \\
\hline & $\eta^{\prime}$ & 0 & 0 & {$\left[0\left(0^{-}\right)\right]$} & $957.78 \pm 0.06$ & $948.2 \pm 99.6$ & $723.4 \pm 126.3$ \\
\hline \multirow{2}{*}{$\begin{array}{l}\text { QED } \\
\text { meson }\end{array}$} & isoscalar & 0 & 0 & {$\left[0\left(0^{-}\right)\right]$} & & $17.9 \pm 1.5$ & $11.2 \pm 1.3$ \\
\hline & isovector & 1 & 0 & {$\left[1\left(0^{-}\right)\right]$} & & $36.4 \pm 3.8$ & $33.6 \pm 3.8$ \\
\hline \multirow{5}{*}{$\begin{array}{c}\text { Possible } \\
\text { QED } \\
\text { meson } \\
\text { candidates }\end{array}$} & $\mathrm{X} 17$ & & & $\left(1^{+}\right) ?$ & $16.70 \pm 0.35 \pm 0.5^{(b)}$ & & \\
\hline & $\mathrm{X} 17$ & & & $\left(0^{-}\right) ?$ & $16.84 \pm 0.16 \pm 0.20^{(c)}$ & & \\
\hline & E38 & & & $?$ & $37.38 \pm 0.71^{(d)}$ & & \\
\hline & E38 & & & $?$ & $40.89 \pm 0.91^{(e)}$ & & \\
\hline & E38 & & & $?$ & $39.71 \pm 0.71^{(f)}$ & & \\
\hline
\end{tabular}

(a): Calibration mass

(b): A. Krasznahorkay et al. $[1],{ }^{8} \mathrm{Be}^{*}$ decay

(c): A. Krasznahorkay et al. [16], ${ }^{4} \mathrm{He}^{*}$ decay

$(d):$ K. Abraamyan et al. [33], $d \mathrm{Cu} \rightarrow \gamma \gamma X$

(e): K. Abraamyan et al. [33], $p \mathrm{Cu} \rightarrow \gamma \gamma X$

$(f)$ : K. Abraamyan et al. [33], $d \mathrm{C} \rightarrow \gamma \gamma X$

Table 1. Comparison of experimental and theoretical masses of neutral, $I_{3}=0$, and $S=0$ QCD and QED mesons obtained with the semi-empirical mass formula (2.37) for QCD mesons and (2.43) for QED mesons, with $\alpha_{\mathrm{QED}}=1 / 137, \alpha_{s}=0.68 \pm 0.08$, and $R_{T}=0.40 \pm 0.04 \mathrm{fm}$.

of 0.6 used for the lowest meson masses in earlier hadron spectroscopy studies [93-96] as a measure of the degree of uncertainties in $\alpha_{s}$, resulting in $\alpha_{s}=0.68 \pm 0.08$.

For the value of $R_{T}$, lattice gauge calculations with dynamical fermions give a flux tube root-mean-square-radius $R_{T}=0.411 \mathrm{fm}$ for a quark-antiquark separation of $0.95 \mathrm{fm}$ [75]. The experimental value of $\left\langle p_{T}^{2}\right\rangle$ of produced hadrons ranges from 0.2 to $0.3 \mathrm{GeV}^{2}$ for $e^{+}$$e^{-}$annihilations at $\sqrt{s}$ from $29 \mathrm{GeV}$ to $90 \mathrm{GeV}$ [83], corresponding to a flux tube radius $R_{T}=\hbar / \sqrt{\left\langle p_{T}^{2}\right\rangle}$ of 0.36 to $0.44 \mathrm{fm}$. It is reasonable to consider flux tube radius parameter to be $R_{T}=0.4 \pm 0.04 \mathrm{fm}$. This set of parameters of $\alpha_{s}=0.68 \pm 0.08$ and $R_{T}=0.40 \pm 0.04$ $\mathrm{fm}$ give an adequate description of the $\pi^{0}, \eta$ and $\eta^{\prime}$ masses as shown in table I.

From our comparison of the experimental and theoretical masses in table 1, we find that by using the method of bosonization and including the confining interaction and the quark condensate, the mass formula (2.37) in the $1+1$ dimensional open string model can indeed describe adequately the masses of $\pi^{0}, \eta$, and $\eta^{\prime}$, approximately within the limits of the uncertainties of the theory. The formulation can be used to extrapolate to the unknown region of open string $q \bar{q}$ QED mesons.

In order to infer the importance of the second quark condensate term relative to the massless quark limit arising from the confining interaction in (2.37), we tabulate in table I the results of the hadron mass values obtained in the massless quark limit. We observe that for the pion mass, the massless quark limit is zero, and the pion mass arises only from the 
second quark condensate term. The importance of the quark condensate diminishes as the hadron masses increases to $\eta$ and $\eta^{\prime}$. Thus, in experiments in which the quark condensate may be affected by the environment in which the hadrons are produced, as for example in a hot quark gluon plasma at various temperatures, the degree of quark condensation may decrease as the temperature increases, resulting in a downward shift of the hadron mass towards the massless quark limit. The massless quark limit may be reached at a critical temperature when the chiral symmetry is fully restored with the absence of a quark condensate. As a consequence, the shift in the hadron masses may be a signature of the restoration of chiral symmetry. It is interesting to note in the QCD meson case that the absence of the quark condensate leads to the pions acquiring a zero mass, which is an indication of the dissociation of the pion into a massless quark and antiquark pair when chiral symmetry is restored. It is reasonable to consider this to be an indication that the occurrence of chiral symmetry restoration for a pion occur alongside with the occurrence of the deconfinement of the quark and the antiquark in the pion.

\subsection{Open string description of the QED mesons}

Having confirmed the approximate validity of the open string description of QCD mesons in $1+1$ dimensions, we proceed to extrapolate to the unknown region of the open string $q \bar{q}$ QED mesons. In this subsection (2.5), we restrict ourselves to the QED interaction with the interaction label superscript set implicitly to $\lambda=0$ for QED. The mass scale in (1.4) gives $m^{\mathrm{QED}} \sim 47 \mathrm{MeV} \gg m_{u}, m_{d}$, but $m^{\mathrm{QED}}$ is comparable to $m_{s}$. In the treatment of QED mesons, it is only necessary to include $u$ and $d$ quarks and antiquarks, with $N_{f}=2$.

We denote flavor states $\phi_{1}=|u \bar{u}\rangle, \phi_{2}=|d \bar{d}\rangle$, and construct the physical isoscalar $\left|\Phi_{1}^{\mathrm{QED}}\right\rangle$ and the isovector $\left|\Phi_{2}^{\mathrm{QED}}\right\rangle$ states as

$$
\begin{aligned}
& \left.\mid(\text { isoscalar }) I=0, I_{3}=0\right\rangle=\Phi_{1}=\left(\phi_{1}+\phi_{2}\right) / \sqrt{2}, \\
& \left.\mid(\text { isovector }) I=1, I_{3}=0\right\rangle=\Phi_{2}=\left(\phi_{1}-\phi_{2}\right) / \sqrt{2} .
\end{aligned}
$$

They are related by $\Phi_{i}=\sum_{f} D_{i f} \phi_{f}$ and $\phi_{i}=\sum_{f} D_{i f} \Phi_{i}$,

$$
\left(\begin{array}{l}
\Phi_{1} \\
\Phi_{2}
\end{array}\right)=\left(\begin{array}{c}
\frac{1}{\sqrt{2}}+\frac{1}{\sqrt{2}} \\
\frac{1}{\sqrt{2}}-\frac{1}{\sqrt{2}}
\end{array}\right)\left(\begin{array}{l}
\phi_{1} \\
\phi_{2}
\end{array}\right), \quad\left(\begin{array}{l}
\phi_{1} \\
\phi_{2}
\end{array}\right)=\left(\begin{array}{c}
\frac{1}{\sqrt{2}}+\frac{1}{\sqrt{2}} \\
\frac{1}{\sqrt{2}}-\frac{1}{\sqrt{2}}
\end{array}\right)\left(\begin{array}{l}
\phi_{1} \\
\phi_{2}
\end{array}\right) .
$$

The mass formula (2.30) for the mass of $\Phi_{i}$ becomes

$$
m_{I}^{2}=\left[\frac{Q_{u}+(-1)^{I} Q_{d}}{\sqrt{2}}\right]^{2} \frac{4 \alpha_{\mathrm{QED}}}{\pi R_{T}^{2}}+m_{u d} e^{\gamma} \mu^{\mathrm{QED}},
$$

where the mass scale $\mu^{\text {QED }}$ for QED mesons is not known. From the results for QCD mesons in (2.33)-(2.35), we expect an analogous relationship relating the mass scale $\mu^{\text {QED }}$ and the quark condensate for QED mesons,

$$
e^{\gamma} \mu^{\mathrm{QED}} \propto|\langle 0|\bar{q} q| 0\rangle|_{\mathrm{QED}},
$$

where $|\langle 0|\bar{q} q| 0\rangle|_{\mathrm{QED}}$ is the quark condensate in the presence of QED gauge interactions between the quark and the antiquark. At the present stage of our development, there is 
no experimental information to quantify $|\langle 0|\bar{q} q| 0\rangle|_{\text {QED }}$. It is also not known whether such a quark condensate term may be affected by the environment such as the temperature or the mechanism of QED meson production. It is only known that for QCD mesons, such a quark condensate term is related to the pion mass. We expect that the quark condensate term should depend on the coupling constant $g_{2 \mathrm{D}}$ of the gauge interaction, whether it be QCD or QED. We note that the first term in (2.30) depends on the coupling constant as $\left(g_{2 \mathrm{D}}\right)^{2}$, and in our comparison in QCD, the quark condensate term is just as important as the massless quark limit for the lightest QCD meson. Thus, pending future amendments, we assume that the second quark condensate term in (2.40) for QED is of order $\left(g_{2 \mathrm{D}}^{\mathrm{QED}}\right)^{2}$, the same as the first massless quark limit term. In this case, we have

$$
\frac{\left(\mu^{\mathrm{QED}} \text { in the quark condensate term for QED }\right)}{\left(\mu^{\mathrm{QCD}} \text { in the quark condensate term for QCD }\right)}=\frac{\left(g_{2 \mathrm{D}}^{\mathrm{QED}}\right)^{2}}{\left(g_{2 \mathrm{D}}^{\mathrm{QCD}}\right)^{2}}=\frac{\alpha_{\mathrm{QED}}}{\alpha_{s}} .
$$

From eqs. (2.33), (2.40), and (2.42), it is then reasonable to consider the phenomenological semi-empirical mass formula for QED mesons as

$$
m_{I}^{2}=\left[\frac{Q_{u}+(-1)^{I} Q_{d}}{\sqrt{2}}\right]^{2} \frac{4 \alpha_{\mathrm{QED}}}{\pi R_{T}^{2}}+m_{\pi}^{2} \frac{\alpha_{\mathrm{QED}}}{\alpha_{s}} .
$$

Here, the first term is the massless quark limit arising from the confining interaction between the quark and the antiquark, with $Q_{u}=2 / 3, Q_{d}=-1 / 3$, and $\alpha_{\mathrm{QED}}=1 / 137$. It depends only on the flux tube radius $R_{T}$. The second term arises from the quark masses and the quark condensate in the presence of the QED interaction.

In applying the above mass formula for QED2 mesons, we extrapolate from the QCD sector to the QED sector by using those $R_{T}$ and $\alpha_{s}$ parameters that describe well the $\pi^{0}$, $\eta$ and $\eta^{\prime}$ QCD mesons in section 2.4. We list the theoretical masses of the neutral, $I_{3}=0$, $S=0$ QED mesons obtained by eq. (2.43) in table I. We find an $I=0$ isoscalar QED meson at $m_{\text {isoscalar }}^{\mathrm{QED}}=17.9 \pm 1.5 \mathrm{MeV}$ and an $\left(I=1, I_{3}=0\right)$ isovector QED meson at $m_{\text {isovector }}^{\text {QED }}=36.4 \pm 3.8 \mathrm{MeV}$. As the $I^{G}\left(J^{P C}\right)$ quantum numbers of the QCD mesons are known, we can infer the quantum numbers of the corresponding QED mesons with the same $I$ and $S$ by analogy. Such an inference by analogy provides a useful tool to determine the quantum numbers and some electromagnetic decay properties of QED mesons. Using such a tool, we find that the isoscalar QED meson has quantum numbers $I^{G}\left(J^{P C}\right)=1^{-}\left(0^{-+}\right)$and the isovector $I_{3}=0$ QED meson has quantum numbers $I^{G}\left(J^{P C}\right)=0^{+}\left(0^{-+}\right)$. Within the theoretical and experimental uncertainties, the matching of the $I\left(J^{\pi}\right)$ quantum numbers and the mass may make the isoscalar QED meson a good candidate for the X17 particle emitted in the decay of the $0\left(0^{-}\right)$state of $\mathrm{He}^{4}$ in [16].

It is interesting to note that for the $I_{3}=0, S=0$ QCD and QED mesons, the mass ordering for the isovector and isoscalar QCD mesons is the reverse of that for the isovector and isoscalar QED mesons. This arises because in QCD mesons there is no difference in the magnitudes and the signs of the color charges of the $u$ and $d$ quarks, with $Q_{u}^{\mathrm{QCD}}=Q_{d}^{\mathrm{QCD}}=1$, whereas in QED mesons there is a difference in the magnitudes and the signs of the electric charges of the $u$ and $d$ quarks, with $Q_{u}^{\mathrm{QED}}=2 / 3$ and $Q_{d}^{\mathrm{QED}}=-1 / 3$. 
In order to show the effects of the second quark condensate term relative to the massless quark climit in (2.43), we tabulate in table I the results of the QED meson masses values obtained in the massless quark limit. One observes that the mass of the isoscalar QED meson with the quark condensate is $17.9 \pm 1.5 \mathrm{MeV}$ but it is reduced to $11.2 \pm 1.3 \mathrm{MeV}$ in the massless quark limit without the quark condensate. The QED meson masses may be affected by the environment in which the mesons are produced, as for example in a hot quark gluon plasma at various temperatures. The degree of QED quark condensation is expected to decrease as the temperature increases, resulting in a downward shift of the meson mass towards the massless quark limit as the temperature increases. The massless quark limit may be reached at a critical temperature when the spontaneously broken chiral symmetry is fully restored with the absence of a quark condensate. When that happens, the mass of the isoscalar QED meson may be shifted to a lower value of about $11.2 \pm 1.3 \mathrm{MeV}$. As a consequence, the shift in the hadron masses may be a signature of the restoration of chiral symmetry. The quark condensate may also be affected by the mechanism for the production of the QED mesons. If an isoscalar QED meson is produced in a mode without a quark condensate, then its mass may be shifted downward to $11.2 \pm 1.3 \mathrm{MeV}$. It is interesting to note that in the QED meson case the absence of the quark condensate leads to a decrease of the isoscalar QED meson mass from $17.9 \pm 1.5 \mathrm{MeV}$ to $11.2 \pm 1.3 \mathrm{MeV}$, but the mass remains non-zero at $11.2 \pm 1.3 \mathrm{MeV}$, indicating that the QED confinement between the quark and the antiquark remains operational even when chiral symmetry is restored. It is reasonable to consider then the possibility that the occurrence of chiral symmetry restoration and the occurrence of the deconfinement of an isoscalar QED meson take place at different temperatures of the environment.

In table 1, we have also listed the hypothetical X17 particle observed in $[1,16]$ and the hypothetical E38 particle observed in $[32,33]$ as possible QED meson candidates because their measured invariant masses appear to be close to the masses of the predicted isoscalar and isovector QED mesons, respectively. It will be of great interest to confirm or refute the existence these hypothetical particles by independent experimental investigations, as a test of the QED meson concepts.

\section{Production of the QCD and QED mesons}

How are QCD and QED mesons produced? We can consider the interaction of particles $A$ and $B$ in figure 1, where $A$ or $B$ can be a nucleon in free space, a nucleon inside a nucleus, or a meson. The double solid lines in figure 1 represent a diquark in the case of a nucleon, or an antiquark in the case of a meson. The production of QCD and QED mesons $C_{i}$ and $D_{i}$ by the interaction of particles $A$ and $B$ may be described by Feynman Diagrams 1(a), 1(b), and 1(c). Diagram 1(a) is for low energies, Diagram 1(b) is for intermediate energies, and Diagram 1(c) is for high energies. The produced particles may be either QCD or QED mesons if the available energy is above the QCD meson mass threshold. On the other hand, if the available energy is below the pion mass threshold, as in the decay of the excited ${ }^{4} \mathrm{He}$ and ${ }^{8} \mathrm{Be}$ in $[1,16]$, the produced particle $C_{1}$ in Diagram 1(a) can only be a QED meson. Diagrams 1(b) and 1(c) may describe the simultaneous production of QCD mesons 


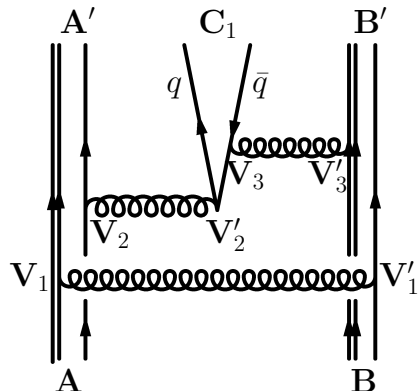

(a)

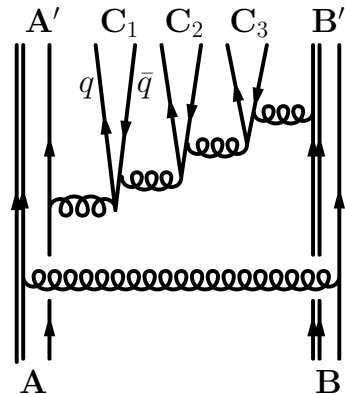

(b)

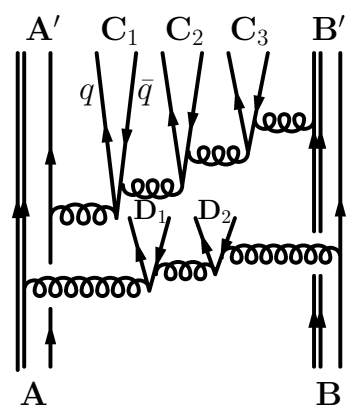

(c)

Figure 1. Feynman diagrams for the production of QCD and QED mesons: (a) $A+B \rightarrow A^{\prime}+$ $B^{\prime}+C_{1}$ at low energies, $(b) A+B \rightarrow A^{\prime}+B^{\prime}+C_{1}+C_{2}+C_{3} \ldots$ at intermediate energies, and $(c)$ $A+B \rightarrow A^{\prime}+B^{\prime}+C_{1}+C_{2}+C_{3}+\ldots+D_{1}+D_{2}+\ldots$ at high energies. The double lines represent a diquark in the case of a baryon and an antiquark in the case of a meson.

and QED mesons in the anomalous soft photon phenomenon in high-energy hadron-proton reactions, when a fraction of the produced particles $C_{i}$ and $D_{i}$ are QED mesons while the dominant fraction are QCD mesons. The produced QED mesons subsequently decay into $e^{+} e^{-}$pairs as anomalous soft photons in [24-27] or as $\gamma \gamma$ pairs in [32, 33, 55-58].

To describe the decay of the excited $0\left(0^{-}\right)$state of ${ }^{4} \mathrm{He}$ at an excitation energy of $21.02 \mathrm{MeV}$, we can consider the preparation of the excited state of ${ }^{4} \mathrm{He}$ by pulling a proton out of a tightly bound ${ }^{4} \mathrm{He}$ nucleus, in the configuration of a stretched string, with the proton and the remainder ${ }^{3} \mathrm{H}^{*}$ at the two ends of the string. We can represent the outside proton as the particle $A$ in Diagram 1(a), and a nucleon in the much heavier ${ }^{3} \mathrm{H}^{*}$ core remainder as the particle $B$ in Diagram 1(a). The strong binding (of $21.01 \mathrm{MeV}$ ) between the proton and the core remainder leads to a strong interaction that polarizes the spatial region between them in the stretched string, leading to the vacuum polarization and the creation of a $q \bar{q}$ pairs at the vertex $V_{2}^{\prime}$ in Diagram 1(a). The creation of the quark and antiquark pair is energetically possible because of the masses of the up and down quarks are only of order a few $\mathrm{MeV}$ [117]. The available energy of $21.01 \mathrm{MeV}$ is above the $q \bar{q}$ pair production threshold but below the pion mass threshold. The produced $q \bar{q}$ pair can only interact with QED interactions to form a QED meson at the appropriate energy. By way of such pair production in vacuum polarization, there may be the occasional production of a quantized open string isoscalar $q \bar{q}$ QED meson $C_{1}$ with the proper quantum numbers at the appropriate QED meson energies. Similarly we can envisage the possible production of $\mathrm{X} 17$ from the $18.15 \mathrm{MeV} 0\left(1^{+}\right)$excited state of ${ }^{8} \mathrm{Be}$ that is prepared by stretching out a proton from one of the two ${ }^{4} \mathrm{He}$ nuclei in the ${ }^{8} \mathrm{Be}$ nucleus. Vacuum polarization with the production of a $q \bar{q}$ pairs may lead to the production of the open string isoscalar $0\left(0^{-}\right)$ QED meson, coming out in the $l=1$ partial wave.

All of the initial and final states of the reaction $(A B) \rightarrow\left(A^{\prime} B^{\prime}\right)+C_{1}$ in Diagram 1(a) involve colorless particles, and it is instructive to follow the color flow of the intermediate states to see how such a reaction proceeds from colorless initial particles $A$ and $B$ to colorless final particles $A^{\prime}, B^{\prime}$ and $C_{1}$ in Diagram 1(a). After the emission of the gluon at the point $V_{1}$ from the diquark of nucleon $A$, the intermediate state of $A$ acquires a color, 
which is however bleached to become the colorless $A^{\prime}$ upon the emission of the gluon at $V_{2}$. Similarly, the nucleon $B$ becomes colored upon absorbing a gluon at vertex $V_{1}^{\prime}$, but the color is bleached upon the absorption of a gluon at $V_{3}^{\prime}$ to lead to the final colorless nucleon $B^{\prime}$. The produced $q \bar{q}$ pair is initially colored because it originates from the gluon at vertex $V_{2}^{\prime}$. The color of the $q \bar{q}$ pair is bleached to become the colorless final state $C_{1}$ upon the emission of a gluon at $V_{3}$. The important ingredient for the occurrence of such a reaction process is the availability of the excitation energy and the small values of the up and down quark masses which facilitate the production of the $q \bar{q}$ pair.

Recently, a boson particle with invariant a mass of about $38 \mathrm{MeV}$ (labeled as the hypothetical E38 particle) decaying into $\gamma \gamma$ pairs has been observed at Dubna in highenergy $\{p \mathrm{C}, d \mathrm{C}, d \mathrm{Cu}\} \rightarrow \gamma \gamma X$ collisions at proton and deuteron incident energies of 5.5, 2.76 , and $3.83 \mathrm{GeV}$ per nucleon, respectively $[32,33]$. The observed E38 mass coincides, within the experimental and theoretical uncertainties, with the predicted mass of $36.4 \pm 3.8$ $\mathrm{MeV}$ for the isovector $\left[I\left(J^{\pi}\right)=0\left(0^{-}\right), I_{3}=0\right]$ QED meson, making the isovector QED meson a good candidate for the E38 particle. Diagrams 1(b) or 1(c), in which one of the final particles is the isovector QED meson, may describe the production of the E38 particle in these high-energy reactions.

In another set of experiments, the raw data of the $\gamma \gamma$ invariant mass spectra in exclusive measurements in $p p \rightarrow \rightarrow p\left(\pi^{+} \pi^{-} \gamma \gamma\right) p[55,56]$ and $\pi^{-} p \rightarrow \pi^{-}\left(\pi^{+} \pi^{-} \gamma \gamma\right) p$ reactions at $p_{\text {lab }}=193 \mathrm{GeV} / \mathrm{c}$ at COMPASS $[57,58]$ exhibit structures at around 10-15 MeV and 38 $\mathrm{MeV}$. It has been argued that these structures may arise from experimental artifacts [59]. On the other hand, it has also been suggested that even after the background data are subtracted, there may remain a significant structure at $38 \mathrm{MeV}$ [60]. What may be of great interest are the following experimental facts: (i) the locations of the energies of these structures at $10-15 \mathrm{MeV}$ and $38 \mathrm{MeV}$ fall within the vicinity of the predicted masses of the isoscalar QED meson and isovector QED meson respectively, (ii) the peak at around 10-15 $\mathrm{MeV}$ in the $\gamma \gamma$ invariant mass spectrum in $p p \rightarrow p\left(\pi^{+} \pi^{-} \gamma \gamma\right) p[55,56]$ appears to be substantially above the neighboring background distribution [55, 56], (iii) diphoton resonance at $38 \mathrm{MeV}$ has been observed in high energy $\{p \mathrm{C}, d \mathrm{C}, d \mathrm{Cu}\} \rightarrow \gamma \gamma X$ reactions [32, 33], and (iv) the occurrence of the structure appears in both $p p$ and $\pi^{-} p$ collisions, indicating that the phenomenon may be more general than a single projectile-target combination. If one looks at the $p p$ and $\pi^{-} p$ reactions at COMPASS from the theoretical viewpoint of Diagram 1(b), it is possible that while most of the produced $q \bar{q}$ pairs in the set of $\left(C_{1}, C_{2}, C_{3}\right)$ are QCD mesons, it cannot be excluded that some of the produced $C_{i}$ particles among the set of three $C_{i}$ particles may be an isoscalar or isovector QED meson with a mass of order $17 \mathrm{MeV}$ or $38 \mathrm{MeV}$. Whether these structures at $10-15 \mathrm{MeV}$ and $38 \mathrm{MeV}$ represent genuine particle states remains a subject for further studies. Future investigations in the region of low $\gamma \gamma$ invariant masses will provide additional tests to confirm or refute the proposed concept of the open string QED mesons.

\section{Transverse momentum distribution of anomalous soft photons}

We would like to examine how the production of quantized QED mesons may be consistent with the anomalous soft photon phenomenon. In figure 2 , the solid circular points 

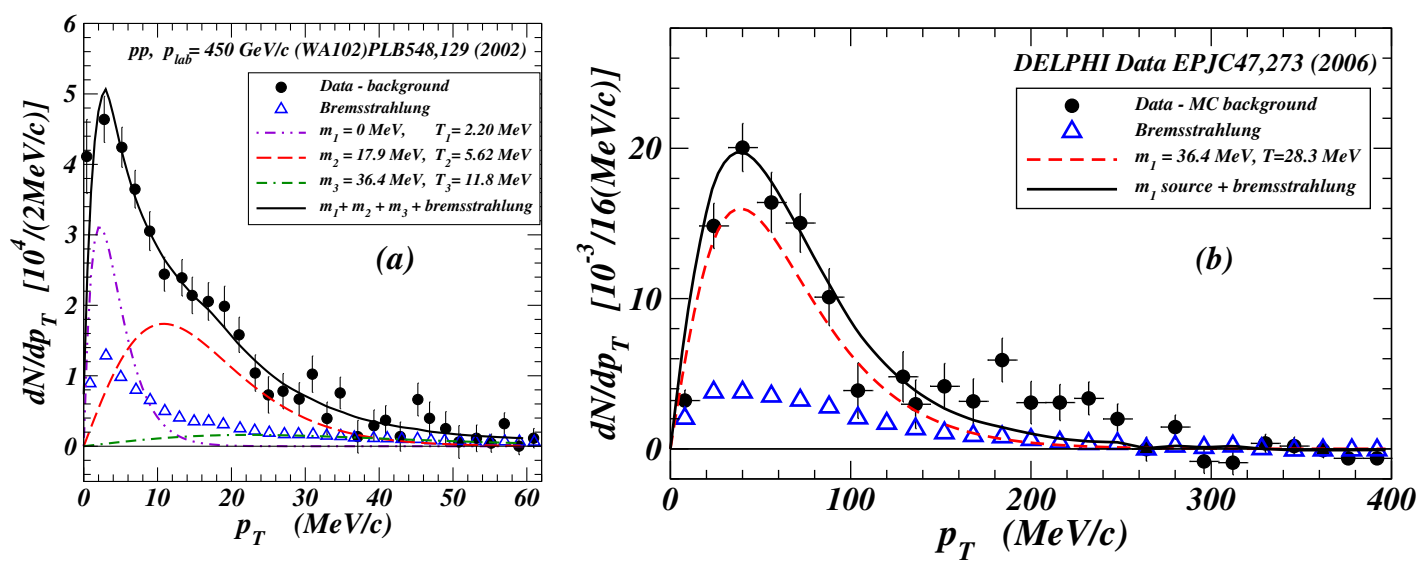

Figure 2. (a) Anomalous soft photon $d N / d p_{T}$ data from $p p$ collisions at $p_{\text {lab }}=450 \mathrm{GeV} /$ c obtained by Belogianni et al. [27]. (b) Anomalous soft photon $d N / d p_{T}$ data from the DELPHI Collaboration for $e^{+} e^{-}$annihilation at the $Z_{0}$ mass of $91.18 \mathrm{GeV}[28]$. The solid circular points represent the experimental data after subtracting the experimental background, and triangle points represent the deduced bremsstrahlung contributions. The total theoretical yields in the thermal model from produced bosons and the additional bremsstrahlung contributions are shown as solid curves. The component yields from different masses of the thermal model are shown as separate curves.

give the experimental $d N / d p_{T}$ data of soft photons measured as $e^{+} e^{-}$pairs after subtracting the experimental background. figure 2(a) is the WA102 results for $p p$ collisions at $p_{\text {lab }}=450 \mathrm{GeV} / \mathrm{c}$ from Belogianni et al. [27], and figure 2(b) is the DELPHI results for $e^{+} e^{-}$annihilation at the $Z_{0}$ mass of $91.18 \mathrm{GeV}$ [28]. They are exclusive measurements in which the momenta of all participating charged particles are measured. The knowledge of the momenta of all initial and final charged particles allows an accurate determination of the bremsstrahlung $d N / d p_{T}$ distributions shown as triangular points in figure (2). As shown in figure 2, the observed yields of soft photons exceed the bremsstrhlung contributions substantially in both $p p$ collisions in 2(a) and $e^{+} e^{-}$annihilations in 2(b). The excess soft photons as $e^{+} e^{-}$pairs constitute the soft photon anomaly that is the subject of our attention.

We would like to inquire how the production of the QED mesons may be consistent with the excess $e^{+} e^{-}$pairs in the anomalous soft photon phenomenon. For such an investigation, we rely on the thermal model which describes well the transverse momentum distributions in the production of hadrons of different masses in high-energy $p p$ collisions [118-121]. We shall assume that the validity of the thermal model can be extended from the production of QCD mesons to the production of QED mesons whose decay products are assumed to appear as anomalous soft photons. In such a thermal model, the transverse momentum distribution of the produced QED mesons is related to the produced QED meson mass $m$ by $[118]$

$$
\frac{d N}{p_{T} d p_{T}}=A e^{-\sqrt{m^{2}+p_{T}^{2}} / T} .
$$

The contribution to the total $d N / d p_{T}$ from each boson of mass $m$ is proportional to 
$p_{T} e^{-\sqrt{m^{2}+p_{T}^{2}} / T}$ which is zero at $p_{T}=0$ and has a peak at the location $p_{T}$ given by

$$
p_{T}^{2}=\frac{1}{2}\left[T^{2}+\sqrt{T^{4}+4 T^{2} m^{2}}\right] .
$$

If $m=0$, then $d N / d p_{T}$ peaks at $p_{T}=T$. If $m$ is much greater than $T$, then $d N / d p_{T}$ peaks at $p_{T} \sim \sqrt{m T}$. Hence, for each contributing boson mass component, the thermal model gives a distribution that starts at zero at $p_{T}=0$ and reaches a peak of $d N / d p_{T}$. The total $d N / d p_{T}$ is a sum of contributions from different QED mesons and boson components,

$$
\frac{d N}{p_{T} d p_{T}}=\sum_{i} A_{i} e^{-\sqrt{m_{i}^{2}+p_{T}^{2}} / T_{i}}
$$

There can be as many contributing bosons as the number of underlying peaks in the $d N / d p_{T}$ spectrum. While different decompositions of the spectrum into different masses (and peaks) are possible, the structure of the $d N / d p_{T}$ data appears to require many components in figure 1(a) and only a single component in figure 2(b). In the thermal model analysis of the $p p$ data in figure 2(a), we note that there appears to be a boson component of real photons with $m_{1}=0$. Because of the $m_{i} T_{i}$ ambiguity ${ }^{3}$ associated with the product of the meson mass $m_{i}$ and the temperature $T_{i}$, we are content with only a consistency analysis. We assume that QED isoscalar and isovector mesons with masses as given by table I are produced in the collision, and their subsequent decay into $e^{+} e^{-}$pairs give rise to the excess $e^{+} e^{-}$pairs observed as anomalous soft photons. Allowing other parameters to vary, the thermal model fit in figure $2(\mathrm{a})$ is obtained with parameters $A_{1}=3.85 \times 10^{4} /(2 \mathrm{MeV} / c)$, $T_{1}=2.20 \mathrm{MeV}, A_{2}=6.65 \times 10^{4} /\left(2(\mathrm{MeV} / c), T_{2}=5.62 \mathrm{MeV}, A_{3}=0.266 \times 10^{4} /(2(\mathrm{MeV} / c)\right.$, and $T_{3}=11.8 \mathrm{MeV}$, where the different components also shown as separate curves. Adding the contributions from the three components onto the bremsstrahlung contributions yields the total $d N / d p_{T}$ shown as the solid curve. The comparison in figure 2(a) indicates that the $p p$ data are consistent with a photon component and a boson component with a mass around $17 \mathrm{MeV}$. The magnitude of the $m_{3}=36.4 \mathrm{MeV}$ component is of the same order as the bremsstrahlung contribution or the noise level, and is rather uncertain. In figure 2(b), the addition of the single component with $A_{1}=2.7 \times 10^{-3} /(16 \mathrm{MeV} / \mathrm{c}), m_{1}=36.4 \mathrm{MeV}$, and $T_{1}=28.3 \mathrm{MeV}$ onto the bremsstrahlung contributions gives a consistent description of the soft photon data in $e^{+} e^{-}$annihilations as shown as the solid curve.

The component with $m_{1}=0$ in figure 2(a) may be associated with the decay of the QED mesons into two photons. If so, it will be of interest to measure the $\gamma \gamma$ invariant mass to look for diphoton resonances, as carried out in [32, 33, 55-58]. The $m_{2}=17.9$ $\mathrm{MeV}$ components in figure 2(a) and the $m_{3}=36.4 \mathrm{MeV}$ component in figure 2(b) may be associated with the predicted isoscalar and isovector QED mesons of table 1. If so, a measurement on the invariant masses of the $m_{2}$ and $m_{3}$ components will be of great interest to confirm the existence of these QED mesons. The recent reports of the observation of a hypothetical E38 boson at $38 \mathrm{MeV}$ and the structures in the $\gamma \gamma$ invariant masses at 10-15 $\mathrm{MeV}$ and $38 \mathrm{MeV}[32,33,55-58]$ provide encouraging impetus for further studies.

\footnotetext{
${ }^{3}$ In fitting the thermal model, the $m_{i} T_{i}$ ambiguity gives different values of $m_{i}$ for different values of $T_{i}$ without changing significantly the overall quality of the fitting.
} 
We can envisage how QED mesons may be produced alongside with QCD mesons in the anomalous soft photon phenomenon in high-energy particle collisions [23, 24, 24-31] in which meson production has been well understood as a string fragmentation process [6772]. Many $q \bar{q}$ strings may be produced when the string joining a valence quark and a valence antiquark are pulled apart. Because quarks and antiquarks interact with both QCD and QED interactions and they may form meson states, both QCD mesons and QED mesons may be simultaneously produced during the high-energy string fragmentation process. Such a simultaneous production of QCD mesons and QED mesons can be alternatively described by the production mechanism of Diagrams 1(b) and 1(c), in which the final produced particles $C_{i}$ or $D_{i}$ may be QCD or QED mesons. The produced QED mesons subsequently decay into $e^{+} e^{-}$and $\gamma \gamma$ pairs which may appear as excess $e^{+} e^{-}$and $\gamma \gamma$ yields to accompany the produced QCD mesons [30,31, 34]. The QED mesons and their decay $e^{+} e^{-}$products will not be produced when hadrons are not produced in $e^{+}+$ $e^{-} \rightarrow \mu^{+}+\mu^{-}$bremsstrahlung [29].

There remain many unresolved questions and uncertainties regarding the anomalous soft photons as presented in figure 2. The thermal model analysis only provides an approximate hint on the possible contributions from many mass components, as the fitting of the boson masses in the thermal model contains ambiguities associated the determination of the masses and temperatures. It is also not known why the two components of $m_{1}=0$ and $m_{1}=16.9 \mathrm{MeV}$ in the $p p$ measurement in figure $1(\mathrm{a})$ are not present in $e^{+} e^{-}$annihilation DELPHI measurement in figure 1(b). What may be important however are the cross section enhancements in many transverse momentum regions, suggesting possible masses where quantized boson masses may occur. The additional measurements of the invariant masses of the excess $e^{+} e^{-}$and $\gamma \gamma$ pairs of the decay photon energies in the neighborhood of these enhancements will reveal whether or not the excess $e^{+} e^{-}$and $\gamma \gamma$ pairs represent quantized bosons, to test the concepts of the QED mesons.

\section{Behavior of a massive QED meson assembly}

The QCD and QED mesons with massless quarks in $1+1$ dimensions cannot decay as the quark and the antiquark execute yo-yo motion along the string. As the string is an idealization of a flux tube, the structure of the flux tube must be taken into account in the physical processes in $3+1$ dimensions. The quark and the antiquark at different transverse coordinates in the tube traveling from opposing longitudinal directions can make a sharp change of their trajectories turning to the transverse direction where the quark and the antiquark can meet and annihilate, leading to the emission of two photons at the vertices $V_{1}$ and $V_{2}$ as depicted in figure 3(a). The coupling of these photons to an electron pair as shown in figure 3(b) leads further to the decay of the QED meson into an electron-positron pair. Thus, by the consideration of the transverse structure of the flux tube, the QED mesons can decay into photons and electron-positron pairs in $3+1$ dimensions.

An astrophysical object consisting of a large assembly of isoscalar $0\left(0^{-}\right)$QED mesons such as the X17 particle with a mass $m_{X}=17 \mathrm{MeV}$ will be an electron-positron and gamma-ray emitter. If the temperature of such an assembly is low, it can form a Bose- 


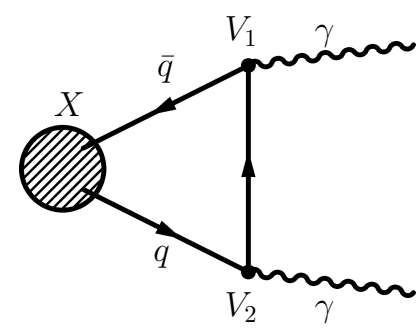

(a)

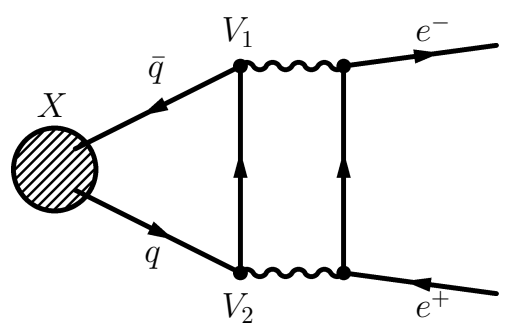

(b)

Figure 3. Decay of the QED meson $\mathrm{X}$ into $(a)$ a $\gamma \gamma$ pair, and $(b)$ an $e^{+} e^{-}$pair.

Einstein condensate. The mode of emission, the emission energies, and the lifetimes depend on the gravitational energy of the assembly. Such assemblies of QED mesons present themselves as good candidates as $e^{+} e^{-}$emitters, gamma-ray emitters, or a part of the primordial cold dark matter. We would like to make estimates on the constraints on masses and radii of such assemblies where they may be found.

We consider an assembly of $A$ number of $m_{X}$ QED mesons of mass $M_{A} \equiv M$ and we place a test QED $m_{X}$ meson at the surface of the assembly at radius $R$, the mass $M_{A+1}$ of the combined system is

$$
M_{A+1}=M_{A}+m_{X}-\frac{G M_{A} m_{X}}{R c^{2}},
$$

where $G$ is the gravitational constant. The $Q$ value for the test QED meson at the surface of the $(A+1)$ assembly to decay into an electron-positron pair is

$$
Q\left((A+1) \rightarrow A+e^{+} e^{-}\right)=m_{X} c^{2}-\frac{G M_{A} m_{X}}{R}-2 m_{e} c^{2},
$$

and the $Q$ value for the test QED meson to decay into two photons is

$$
Q((A+1) \rightarrow A+2 \gamma)=m_{X} c^{2}-\frac{G M_{A} m_{X}}{R} .
$$

Thus, the QED meson $m_{X}$ will not decay into an electron-positron pair when the mass and radius of the assembly satisfy

$$
\frac{M}{R}>\frac{c^{2}}{G}\left(1-\frac{2 m_{e}}{m_{X}}\right),
$$

and the QED meson $m_{X}$ will not decay into two photons nor an $e^{+} e^{-}$pair when $M$ and $R$ satisfy

$$
\frac{M}{R}>\frac{c^{2}}{G} .
$$

Upon using the mass and the radius of the sun as units, it is convenient to define a dimensionless boundary value $B_{0}$ given by

$$
B_{0}=\frac{c^{2} R_{\odot}}{G M_{\odot}}=4.71 \times 10^{5} .
$$

A QED meson assembly will behave differently depending on its $M / R$ values as follows: 
1. The QED meson assembly will emit electron-positron pairs and gamma rays if

$$
B_{0}\left(1-\frac{2 m_{e}}{m_{X}}\right)>\frac{M / M_{\odot}}{R / R_{\odot}}
$$

2. The QED meson assembly will emit only gamma rays but no $e^{+} e^{-}$pairs, if

$$
B_{0}>\frac{M / M_{\odot}}{R / R_{\odot}}>B_{0}\left(1-\frac{2 m_{e}}{m_{X}}\right) .
$$

3. The QED meson assembly will not emit $e^{+} e^{-}$pairs nor gamma rays, if

$$
\frac{M / M_{\odot}}{R / R_{\odot}}>B_{0}
$$

which is essentially the condition for a QED meson black hole.

The above boundaries characterize the properties of QED meson assemblies as $e^{+} e^{-}$emitters, gamma-ray emitters, or dark matter. An assembly of QED mesons satisfying (5.5) (which is the same as or (5.9)) can be a good candidate for a primordial cold blackhole dark matter, as it is non-baryonic, created at the hadronization stage of the quark-gluon plasma phase transition, and not from a stellar collapse.

\section{Conclusions and discussions}

Many interesting questions have been brought into focus with the observations of the X17 particle [1, 16], the E38 particle [32, 33], and the related anomalous soft photon phenomenon [23-31]. Our investigations provide answers to some of these question by linking them together in a coherent framework of open string QED mesons. However, many unanswered questions remain and these questions will require further theoretical and experimental studies. The central questions are (i) whether quarks and antiquarks interacting with the QED interaction can form confined and bound QED mesons in the mass range of many tens of $\mathrm{MeV}$ as suggested in [34], (ii) whether the X17 and E38 particles, and the parent particles of anomalous soft photons are QED mesons, and (iii) whether there can be additional tests to confirm or refute the existence of the open string QED mesons.

On the theoretical side, it can be argued that Schwinger already showed that massless fermions and antifermions interacting with QED interactions in 1+1 dimensions can form confined and bound boson states $[87,88]$. As a quark and an antiquark cannot be isolated and the intrinsic motion of a $q \bar{q}$ system in its lowest-energy states lies predominantly in $1+1$ dimensions [62-72], an open string description in $1+1$ dimensions can be applied to study the $q \bar{q}$ system in QCD and QED interactions. Such interactions lead to confined and bound physical QCD and QED meson states [34-36]. We show that the $\pi^{0}, \eta$, and $\eta^{\prime}$ can be adequately described as open string QCD mesons in 1+1 dimensions, when we properly take into account the relevant physical effects. The extrapolation from the QCD mesons to the QED mesons with $q$ and $\bar{q}$ interacting with QED interactions lead to an isoscalar 
$I\left(J^{\pi}\right)=0\left(0^{-}\right)$QED meson state at $17.9 \pm 1.5 \mathrm{MeV}$ and an isovector $\left(I\left(J^{\pi}\right)=1\left(0^{-}\right), I_{3}=0\right)$ QED meson states at $36.4 \pm 3.8 \mathrm{MeV}$.

On the experimental side, possible occurrence of the QED mesons can be confirmed or refuted by searching for the decay product of $e^{+} e^{-}$and $\gamma \gamma$ pairs. The decay experiments carried out in $[1,16]$ are good examples for such explorations with the observation of the X17 particle at an $e^{+} e^{-}$invariant mass of $17 \mathrm{MeV}$, from the decay of the excited $0\left(0^{-}\right)$state of $\mathrm{He}^{4}$. The matching of the quantum numbers and the masses, within the experimental and theoretical uncertainties, make the isoscalar $0\left(0^{-}\right)$QED meson a good candidate for the X17 particle. There is another E38 MeV particle with a mass of about $38 \mathrm{MeV}$ observed in the $\gamma \gamma$ invariant mass spectrum in high-energy $p \mathrm{C}, d \mathrm{C}, d \mathrm{Cu}$ collisions at Dubna [32,33]. Again, the matching of the predicted mass of the isovector QED meson with the mass of the hypothetical E38 particle, within the experimental and theoretical uncertainties, makes the isovector $1\left(0^{-}\right)$QED meson a good candidate for the E38 particle. There are furthermore possible structures in the $\gamma \gamma$ invariant mass spectra in the raw data at $10-15 \mathrm{MeV}$ and $38 \mathrm{MeV}$ in high-energy $p p \rightarrow p\left(\pi^{+} \pi^{-} \gamma \gamma\right) p$ and $\pi^{-} p \rightarrow \pi^{-}\left(\pi^{+} \pi^{-} \gamma \gamma\right) p$ reactions at COMPASS [55-61]. As the regions of $10-15 \mathrm{MeV}$ and $38 \mathrm{MeV}$ boson masses coincide with the predicted masses of the isoscalar and isovector QED mesons, a careful investigation of this region of low $\gamma \gamma$ invariant masses will be of great interest.

Whether the isoscalar QED meson is related to the $\left(I\left(J^{\pi}\right)=0\left(1^{+}\right)\right.$X17 particle observed in the decay of ${ }^{8} \mathrm{Be}$ remains to be investigated. If the $0\left(1^{+}\right)$excited state of ${ }^{8} \mathrm{Be}$ emits the particle in the $l=1$ partial wave state, then the emitted particle can be a $0\left(0^{-}\right)$ particle, the same as the X17 particle observed in the ${ }^{4} \mathrm{He}$ decay. It will be necessary to check experimentally how the ${ }^{8} \mathrm{Be}$ nucleus in the excited $0\left(1^{+}\right)$state decays. Additional nuclear experiments to confirm ${ }^{8} \mathrm{Be}$ and ${ }^{4} \mathrm{He}$ measurements in $[1,16]$ will also be of interest.

The possible occurrence of the X17 particle and QED mesons receives indirect support from the presence of $e^{+} e^{-}$excesses observed in the anomalous soft photon phenomenon. In particular, the structure of the anomalous soft photon $d N / d p_{T}$ spectrum in the region of $12-20 \mathrm{MeV} / \mathrm{c}$ in high-energy $p p$ collisions at $p_{\mathrm{lab}}=450 \mathrm{GeV} / \mathrm{c}$ [27] is consistent with a possible production of a particle with a mass around $17 \mathrm{MeV}$. The structure of the $d N / d p_{T}$ spectrum in the region of $40 \mathrm{MeV} / \mathrm{c}$ in $e^{+} e^{-}$annihilation at the $Z_{0}$ mass of $91.18 \mathrm{GeV}$ from the DELPHI Collaboration [28] is consistent with a possible production of a particle with a mass around $38 \mathrm{MeV}$. The studies of the X17 and E38 particles and the anomalous soft photons appear to be intimately connected. Other similar searches can be carried out in experiments where hadrons are produced, in high-energy hadron- $p, p p, p A$, and $A A$ collisions as well as high-energy $e^{+} e^{-}$annihilations. The indirect support suggests further needs to study the invariant masses of $e^{+} e^{-}$and $\gamma \gamma$ pairs in the low $p_{T}$ regions.

In our first exploration of the QED mesons, we have limited our studies only to the $S=0, L=0$ and $I_{3}=0$ states. Because of the composite nature of such QED mesons, other collective rotaional and vibrational states of the open string with different $L, S$ and other quantum numbers are also possible. It will be of great interest to extend the frontier of QED mesons into new regions both theoretically and experimentally.

We would like to address the relevance of the QED mesons with regard to the production of dark matter. We envisage that in the early evolution of the universe after 
the big bang, the universe will go through the stage of quark-gluon plasma production with deconfined quarks and gluons. As the primordial matter cools down the quark-gluon plasma undergoes a phase transition from the deconfined phase to the confined phase, and hadronization occurs. Hadrons are then produced from the quark-gluon plasma by way of flux tube production and string fragmentation. As quarks and antiquarks interact with both QCD and QED interactions, the hadron production of QCD mesons will be accompanied by QED meson production, just as the production of hadrons is accompanied by the production of anomalous soft photons in high-energy hadron-nucleon collisions and $e^{+} e^{-}$ annihilations observed experimentally [23, 24, 24-31]. The produced QED mesons are presumably tightly bound and non-interacting in the Schwinger's picture of two-dimensional space-time. In the physical four dimensional space-time with perturbative residual interactions, they can however decay into photons or $e^{+} e^{-}$pairs. Thus, gravitating assemblies of QED mesons are $e^{+} e^{-}$emitters and gamma-ray emitters. On the other hand, if they find themselves in spatial locations where their gravitational binding energies exceed their rest masses, then their decay into photons or electron-positron pairs will be inhibited. There can be QED meson assemblies produced at this stage where the gravitational binding energies of the QED mesons exceed their rest masses. For such QED meson assemblies, the QED mesons will be stable against particle decays and photon emissions. They may form the primordial cold dark matter that may be the source of gravitational attraction for other objects.

The recent inclusive experiment of the NA64 Collaboration [12-14] of finding no "dark" soft photon excesses needs to reconcile with the earlier finding of anomalous soft photon excesses in high energy hadron- $p, p p$, and $e^{+} e^{-}$collisions [23-31]. In the detection of anomalous soft photons in the DELPHI Collaboration [31], the photon detection is carried out by studying the electron and positron tracks in a TPC, while in the NA64 experiment, the produced soft photon needs to penetrate a calorimeter and is detected downstream in a separate calorimeter. It is not known whether the difference in the detection setups and techniques may account for the presence or absence of excess soft photons in the two measurements.

Future work also calls for experimental and theoretical studies of the properties of the X17 and E38 particles and their reactions. Much theoretical work will need to be done to study the decays, the properties, and the reactions of QED mesons in free space and in strong gravitational fields to shed more lights on the fate of the QED meson assembly in the possible primordial dark matter environment.

As it is suggested here that the hadronization at the early history of the universe in the quark-gluon plasma phase generates simultaneously the QED meson assemblies as seeds for primordial dark matter, it will be of great interest to study whether QED mesons as excess $e^{+} e^{-}$and $\gamma \gamma$ pairs with various invariant masses are produced in high-energy heavy-ion collisions where quark gluon plasma may be produced.

\section{Acknowledgments}

The author wishes to thank Prof. Y. Jack Ng for helpful communications and encouragement. The author would like to thank Profs. A. Koshelkin, X. Artru, I. Y. Lee, Gang 
Wang, Xiguang Cao, R. Varner, K. F. Liu, V. Perelpelitsa, S. Sorensen, W. R. Hix, and J. C. Peng for helpful discussions. The research was supported in part by the Division of Nuclear Physics, U.S. Department of Energy under Contract DE-AC05-00OR22725.

Open Access. This article is distributed under the terms of the Creative Commons Attribution License (CC-BY 4.0), which permits any use, distribution and reproduction in any medium, provided the original author(s) and source are credited.

\section{References}

[1] A.J. Krasznahorkay et al., Observation of anomalous internal pair creation in ${ }^{8}$ Be: a possible indication of a light, neutral boson, Phys. Rev. Lett. 116 (2016) 042501 [arXiv:1504.01527] [INSPIRE].

[2] X. Zhang and G.A. Miller, Can nuclear physics explain the anomaly observed in the internal pair production in the Beryllium-8 nucleus?, Phys. Lett. B 773 (2017) 159 [arXiv: 1703.04588] [INSPIRE].

[3] J. Feng et al., Protophobic fifth force interpretation of the observed anomaly in ${ }^{8}$ Be nuclear transitions, Phys. Rev. Lett. 117 (2016) 071803.

[4] J.L. Feng et al., Particle physics models for the $17 \mathrm{MeV}$ anomaly in beryllium nuclear decays, Phys. Rev. D 95 (2017) 035017 [arXiv:1608.03591] [INSPIRE].

[5] B. Fornal, Is there a sign of new physics in beryllium transitions?, Int. J. Mod. Phys. A 32 (2017) 1730020 [arXiv: 1707.09749] [inSPIRE].

[6] NA48/2 collaboration, Search for the dark photon in $\pi^{0}$ decays, Phys. Lett. B 746 (2015) 178 [arXiv: 1504.00607] [INSPIRE].

[7] L. Delle Rose, S. Khalil and S. Moretti, Explanation of the $17 \mathrm{MeV}$ Atomki anomaly in a U(1)'-extended two Higgs doublet model, Phys. Rev. D 96 (2017) 115024 [arXiv: 1704.03436] [INSPIRE].

[8] L. Delle Rose, S. Khalil, S.J.D. King, S. Moretti and A.M. Thabt, Atomki anomaly in family-dependent $\mathrm{U}(1)^{\prime}$ extension of the standard model, Phys. Rev. D 99 (2019) 055022 [arXiv: 1811.07953] [INSPIRE].

[9] U. Ellwanger and S. Moretti, Possible explanation of the electron positron anomaly at 17 MeV in ${ }^{8}$ Be transitions through a light pseudoscalar, JHEP 11 (2016) 039.

[10] D.S.M. Alves and N.J. Weiner, A viable QCD axion in the MeV mass range, JHEP 07 (2018) 092.

[11] M. Munch, O. Sølund Kirsebom, J.A. Swartz, K. Riisager and H.O.U. Fynbo, Measurement of the full excitation spectrum of the ${ }^{7} \mathrm{Li}(p, \gamma) \alpha \alpha$ reaction at $441 \mathrm{keV}$, Phys. Lett. $B \mathbf{7 8 2}$ (2018) 779 [arXiv : 1802.10404] [INSPIRE].

[12] NA64 collaboration, Search for a hypothetical $16.7 \mathrm{MeV}$ gauge boson and dark photons in the NA64 experiment at CERN, Phys. Rev. Lett. 120 (2018) 231802 [arXiv:1803.07748] [INSPIRE].

[13] NA64 collaboration, Search for vector mediator of dark matter production in invisible decay mode, Phys. Rev. D 97 (2018) 072002 [arXiv:1710.00971] [INSPIRE]. 
[14] NA64 collaboration, Improved limits on a hypothetical X(16.7) boson and a dark photon decaying into $e^{+} e^{-}$pairs, Phys. Rev. D 101 (2020) 071101 [arXiv:1912.11389] [InSPIRE].

[15] PADME collaboration, Searching for dark photons with the PADME experiment, in the proceedings of the $19^{\text {th }}$ Frascati Spring School "Bruno Touschek" in Nuclear, Subnuclear and Astroparticle Physics, May 7-11, Frascati, Italy (2018).

[16] A.J. Krasznahorkay et al., New evidence supporting the existence of the hypothetical X17 particle, arXiv:1910:10459.

[17] M. El-Nadi and O.E. Badawy, Production of a new light neutral boson in high-energy collisions, Phys. Rev. Lett. 61 (1988) 1271 [InSPIRE].

[18] M.E. El-Nadi et al., External electron pair production in high-energy collisions, Nuovo Cim. A 109 (1996) 1517.

[19] F.W.N. de Boer et al., A deviation in internal pair conversion, Phys. Lett. B 388 (1996) 235 [INSPIRE].

[20] F. de Boer, Anomalous internal pair conversion signaling elusive light neutral particles, arXiv:0511.04912.

[21] P.L. Jain and G. Singh, Search for new particles decaying into electron pairs of mass below $100 \mathrm{MeV} / \mathrm{c}^{2}$, J. Phys. G 34 (2007) 129.

[22] F.W.N. deBoer and C.A. Fields, A re-evaluation of evidence for light neutral bosons in nuclear emulsions, Int. J. Mod. Phys. E 20 (2011) 1787 [arXiv:1001.3897] [InSPIRE].

[23] P.V. Chliapnikov et al., Observation of direct soft photon production in $K^{+} p$ interactions at $70 \mathrm{GeV} / \mathrm{c}$, Phys. Lett. B 141 (1984) 276 [InSPIRE].

[24] EHS/NA22 collaboration, Direct soft photon production in $K^{+} p$ and $\pi^{+} p$ interactions at $250 \mathrm{GeV} / \mathrm{c}, \mathrm{Z}$. Phys. C 51 (1991) 541 [InSPIRE].

[25] SOPHIE/WA83 collaboration, Observation of direct soft photon production in $\pi^{-} p$ interactions at $280 \mathrm{GeV} / \mathrm{c}$, Phys. Lett. B 305 (1993) 182 [INSPIRE].

[26] WA91 collaboration, Confirmation of a soft photon signal in excess of QED expectations in $\pi^{-} p$ interactions at $280 \mathrm{Gev} / c$, Phys. Lett. B 408 (1997) 487 [hep-ex/9710006] [INSPIRE].

[27] A. Belogianni et al., Observation of a soft photon signal in excess of QED expectations in pp interactions, Phys. Lett. B 548 (2002) 129 [InSPIRE].

[28] DELPHI collaboration, Evidence for an excess of soft photons in hadronic decays of $Z^{0}$, Eur. Phys. J. C 47 (2006) 273 [hep-ex/0604038] [INSPIRE].

[29] DELPHI collaboration, Observation of the muon inner Bremsstrahlung at LEP1, Eur. Phys. J. C 57 (2008) 499 [arXiv:0901.4488] [InSPIRE].

[30] V. Perepelitsa, Anomalous soft photons in hadronic decays of $Z^{0}$, in the proceedings of the XXXIX International Symposium on Multiparticle Dynamics, September 4-9, Gomel, Belarus (2009) [Nonlin. Phenom. Complex Syst. 12 (2009) 343].

[31] DELPHI collaboration, Study of the dependence of direct soft photon production on the jet characteristics in hadronic $Z^{0}$ decays, Eur. Phys. J. C 67 (2010) 343 [arXiv:1004.1587] [INSPIRE].

[32] K. Abraamyan et al., Observation of the $E_{38}$-boson, arXiv:1208.3829 [INSPIRE]. 
[33] K. Abraamyan et al., Check of the structure in photon pairs spectra at the invariant mass of about $38 \mathrm{MeV} / \mathrm{c}^{2}$, EPJ Web Conf. 204 (2019) 08004.

[34] C.-Y. Wong, Anomalous soft photons in hadron production, Phys. Rev. C 81 (2010) 064903 [arXiv: 1001.1691] [INSPIRE].

[35] C.-Y. Wong, Anomalous soft photons associated with hadron production in string fragmentation, AIP Conf. Proc. 1343 (2011) 447 [arXiv:1011.6265] [INSPIRE].

[36] C.-Y. Wong, An overview of the anomalous soft photons in hadron production, talk given at the International Conference on the Structure and the Interactions of the Photon, May 20-24, Paris, France (2014), arXiv: 1404.0040 [INSPIRE].

[37] L. Van Hove, Cold quark-gluon plasma and multiparticle production, Annals Phys. 192 (1989) 66 [inSPIRE].

[38] P. Lichard and L. Van Hove, Cold quark-gluon plasma as source of very soft photons in high-energy collisions, Phys. Lett. B 245 (1990) 605 [INSPIRE].

[39] V. Balek, N. Pisutova and J. Pisut, The puzzle of very soft photon production in hadronic Interactions, Acta. Phys. Pol. B 21 (1990) 149.

[40] S.M. Darbinian, K.A. Ispirian and A.T. Margarian, Unruh radiation of quarks and the soft photon puzzle in hadronic interactions, Sov. J. Nucl. Phys. 54 (1991) 364 [INSPIRE].

[41] W. Czyz and W. Florkowski, Soft photon production in the boost invariant color flux tube model, Z. Phys. C 61 (1994) 171 [INSPIRE].

[42] P. Lichard, Consistency of data on soft photon production in hadronic interactions, Phys. Rev. D 50 (1994) 6824 [hep-ph/9812206] [INSPIRE].

[43] O. Nachtmann, Nonperturbative QCD effects in high-energy collisions, hep-ph/9411345 [INSPIRE].

[44] G.W. Botz, P. Haberl and O. Nachtmann, Soft photons in hadron hadron collisions: synchrotron radiation from the QCD vacuum?, Z. Phys. C 67 (1995) 143 [hep-ph/9410392] [INSPIRE].

[45] E. Kokoulina, A. Kutov and V. Nikitin, Gluon dominance model and cluster production, Braz. J. Phys. 37 (2007) 785.

[46] M. Volkov, E. Kokoulina and E. Kuraev, Gluon dominance model and cluster production, Ukr. J. Phys. 49 (2003) 1252.

[47] Yu.A. Simonov, Di-pion decays of heavy quarkonium in the field correlator method, Phys. Atom. Nucl. 71 (2008) 1049 [hep-ph/07113626].

[48] Yu.A. Simonov, Di-pion emission in heavy quarkonia decays, JETP Lett. 87 (2008) 123.

[49] Yu.A. Simonov and A.I. Veselov, Bottomium $\Upsilon(5 S)$ decays into B $\bar{B}$ and $B \bar{B} \pi$, JETP Lett. $88(2008) 5$.

[50] Y. Simonov and A.I. Veselov, Strong decays and dipion transitions of Upsilon(5S), Phys. Lett. B 671 (2009) 55 [arXiv:0805.4499] [INSPIRE].

[51] Y. Hatta and T. Ueda, Soft photon anomaly and gauge/string duality, Nucl. Phys. B 837 (2010) 22 [arXiv:1002.3452] [INSPIRE].

[52] D.E. Kharzeev and F. Loshaj, Anomalous soft photon production from the induced currents in Dirac sea, Phys. Rev. D 89 (2014) 074053 [arXiv: 1308.2716] [InSPIRE]. 
[53] E. van Beveren and G. Rupp, First indications of the existence of a $38 \mathrm{MeV}$ light scalar boson, arXiv:1102.1863 [INSPIRE].

[54] E. van Beveren and G. Rupp, Material evidence of a 38 MeV boson, arXiv:1202.1739 [INSPIRE].

[55] COMPASS collaboration, Test of OZI violation in vector meson production with COMPASS, in 14th International Conference on Hadron Spectroscopy, 9, 2011 [arXiv: 1109.0272] [INSPIRE].

[56] J. Bernhard, Exclusive vector meson production in pp collisions at the COMPASS experiment, Ph.D. Thesis, University of Mainz, Mainz, Germany (2014).

[57] COMPASS collaboration, The exotic $\eta^{\prime} \pi^{-}$Wave in $190 \mathrm{GeV} \pi^{-} p \rightarrow \pi^{-} \eta^{\prime} p$ at COMPASS, eConf C 110613 (2011) 83 [arXiv:1108.6191] [InSPIRE].

[58] T. Schlüter, The $\pi^{-} \eta$ and and $\pi^{-} \eta^{\prime}$ systems in exclusive $190 \mathrm{GeV} / \mathrm{c} \pi^{-} p$ reactions at COMPASS, Ph.D. Thesis, University of München, München, Germany (2012).

[59] COMPASS collaboration, Comment on "Material evidence of a $38 \mathrm{MeV}$ boson", arXiv: 1204.2349 [INSPIRE].

[60] E. van Beveren and G. Rupp, Reply to comment on "Material evidence of a $38 \mathrm{MeV}$ boson", arXiv: 1204.3287 [INSPIRE].

[61] E. van Beveren and G. Rupp, $Z_{0}(57)$ and $E(38)$ : possible surprises in the Standard Model, accepted for publication in Acta Phys. Pol. B Proc. Suppl., arXiv:2005.08559 [InSPIRE].

[62] G. Veneziano, Construction of a crossing-simmetric, Regge-behaved amplitude for linearly rising trajectories, Nuovo Cim. A $\mathbf{5 7}$ (1968) 190.

[63] Y. Nambu, Quark model of the factorization of the Veneziano Amplitude, in Lectures at the Copenhagen Symposium: Symmetry and Quark Models, R. Chand ed., Gordon and Breach, U.K. (1970).

[64] T. Goto, Relativistic quantum mechanics of one-dimensional mechanical continuum and subsidiary condition of dual resonance model, Prog. Theor. Phys. 46 (1971) 1560.

[65] G. 't Hooft, A planar diagram theory for strong interactions, Nucl. Phys. B 72 (1974) 461 [INSPIRE].

[66] G. 't Hooft, A two-dimensional model for mesons, Nucl. Phys. B 75 (1974) 461 [INSPIRE].

[67] X. Artru and G. Mennessier, String model and multiproduction, Nucl. Phys. B 70 (1974) 93 [INSPIRE].

[68] B. Andersson, G. Gustafson and T. Sjöstrand, A general model for jet fragmentation, Z. Phys. C 20 (1983) 317.

[69] B. Andersson, G. Gustafson, G. Ingelman and T. Sjöstrand, Parton fragmentation and string dynamics, Phys. Rep. 97 (1983) 31.

[70] T. Sjöstrand and M. Bengtsson, The Lund Monte Carlo for jet fragmentation and $e^{+} e^{-}$ physics - Jetset version 6.3 - An update, Comput. Phys. Comm. 43 (1987) 367.

[71] J.D. Bjorken, Lectures presented in the 1973 Proceedings of the Summer Institute on Particle Physics, edited by Zipt, SLAC-167 (1973).

[72] A. Casher, J.B. Kogut and L. Susskind, Vacuum polarization and the absence of free quarks, Phys. Rev. D 10 (1974) 732 [INSPIRE]. 
[73] S. Huang, J.W. Negele and J. Polonyi, Meson structure in QCD in Two-dimensions, Nucl. Phys. B 307 (1988) 669 [INSPIRE].

[74] SESAM collaboration, Observation of string breaking in QCD, Phys. Rev. D 71 (2005) 114513 [hep-lat/0505012] [INSPIRE].

[75] P. Cea, L. Cosmai, F. Cuteri and A. Papa, Flux tubes in QCD with $(2+1)$ HISQ fermions, PoS (LATTICE2016) 344 [arXiv: 1701.03371] [INSPIRE].

[76] C.Y. Wong, R.-C. Wang and C.C. Shih, Study of particle production using two-dimensional bosonized QED, Phys. Rev. D 44 (1991) 257 [InSPIRE].

[77] G. Gatoff and C.Y. Wong, Origin of the soft $p_{T}$ spectra, Phys. Rev. D 46 (1992) 997.

[78] C.Y. Wong and G. Gatoff, The transverse profile of a color flux tube, Phys. Rep. 242 (1994) 489.

[79] C.Y. Wong, Introduction to high-energy heavy-ion collisions, World Scientific, Singapore (1994).

[80] C.-Y. Wong, The Wigner function of produced particles in string fragmentation, Phys. Rev. C 80 (2009) 054917 [arXiv:0903.3879] [INSPIRE].

[81] TPC/Two_Gamma collaboration, Charged hadron production in $e^{+}-e^{-}$annihilation at $s^{1 / 2}=29 \mathrm{GeV}$, Lawrence Berkeley Laboratory Report LBL-23737 (1988).

[82] W. Hofmann, Particle composition in hadronic jets in $e^{+}-e^{-}$annihilation, Ann. Rev. Nucl. Sci. 38 (1988) 279.

[83] A. Petersen et al., Multi-Hadronic Events at $E_{\mathrm{CM}}=29 \mathrm{GeV}$ and predictions of QCD models from $E_{\mathrm{CM}}=29 \mathrm{GeV}$ to $E_{\mathrm{CM}}=93 \mathrm{GeV}$, Phys. Rev. $D 37$ (1988) 1 [inSPIRE].

[84] SLD collaboration, Production of $\pi^{+}, K^{+}, K^{0}, K *^{0}, \phi, p$, and $\Lambda^{0}$ in hadronic $Z^{0}$ decays, Phys. Rev. D 59 (1999) 052001 [hep-ex/9805029] [INSPIRE].

[85] DELPHI collaboration, Energy dependence of inclusive spectra in $e^{+} e^{-}$annihilation, Phys. Lett. B 459 (1999) 397 [INSPIRE].

[86] BRAHMS collaboration, Rapidity densities of $\pi^{ \pm}, K^{ \pm}, p$ and $\bar{p}$ in $p+p$ and $d+A u$ collisions at $\sqrt{s}_{N N}=200 \mathrm{GeV}$, J. Phys. G 35 (2008) 104129 [inSPIRE].

[87] J. Schwinger, Gauge invariance and mass II, Phys. Rev. 128 (1962) 2425 [INSPIRE].

[88] J. Schwinger, Gauge theory of vector particles, Theoretical Physics Trieste Lectures, IAEA, Vienna, Austria (1963) .

[89] H. Georgi, The Schwinger point, JHEP 11 (2019) 057 [arXiv: 1905.09632] [INSPIRE].

[90] H. Georgi and B. Noether, Non-perturbative effects and unparticle physics in generalized Schwinger models, arXiv: 1908.03279 [INSPIRE].

[91] H. Georgi and B. Warner, Generalizations of the Sommerfield and Schwinger models, JHEP 01 (2020) 047 [arXiv: 1907.12705] [INSPIRE].

[92] A.V. Koshelkin and C.-Y. Wong, The compactification of $Q C D_{4}$ to $Q C D_{2}$ in a flux tube, Phys. Rev. D 86 (2012) 125026 [arXiv:1212.3301] [INSPIRE].

[93] C.-Y. Wong, E.S. Swanson and T. Barnes, Cross-sections for $\pi^{-}$and rho induced dissociation of $J / \psi$ and $\psi^{\prime}$, Phys. Rev. C 62 (2000) 045201 [hep-ph/9912431] [INSPIRE]. 
[94] C.-Y. Wong, E.S. Swanson and T. Barnes, Heavy quarkonium dissociation cross-sections in relativistic heavy ion collisions, Phys. Rev. C 65 (2002) 014903 [Erratum ibid. 66 (2002) 029901] [nucl-th/0106067] [INSPIRE].

[95] M. Baldicchi, A.V. Nesterenko, G.M. Prosperi and C. Simolo, QCD coupling below $1 \mathrm{GeV}$ from quarkonium spectrum, Phys. Rev. D 77 (2008) 034013 [arXiv:0705.1695] [INSPIRE].

[96] A. Deur, S.J. Brodsky and G.F. de Teramond, The QCD running coupling, Prog. Part. Nucl. Phys. 90 (2016) 1 [arXiv: 1604.08082] [inSPIRE].

[97] S.R. Coleman, R. Jackiw and L. Susskind, Charge shielding and quark confinement in the massive Schwinger model, Annals Phys. 93 (1975) 267 [INSPIRE].

[98] S.R. Coleman, More about the massive Schwinger model, Annals Phys. 101 (1976) 239 [INSPIRE].

[99] M.B. Halpern, Quantum solitons which are SU(N) Fermions, Phys. Rev. D 12 (1975) 1684 [INSPIRE].

[100] E. Witten, Non-Abelian bosonisation in two dimensions, Commun. Math. Phys. 92 (1984) 455 .

[101] D. Gepner, Nonabelian bosonization and multiflavor QED and QCD in two-dimensions, Nucl. Phys. B 252 (1985) 481 [inSPIRE].

[102] J. Ellis, Y. Frishman, A. Hanany and M. Karliner, Quark solitons as constituents of hadrons, Nucl. Phys. B 382 (1992) 189212.

[103] Y. Frishman and J. Sonnenschein, Bosonization and QCD in two dimensions, Phys. Rep. 223 (1993) 309.

[104] C.-Y. Wong, R.-C. Wang and J.-S. Wu, Schwinger particle production mechanism for a finite length flux tube with transverse confinement, Phys. Rev. D 51 (1995) 3940 [hep-ph/9412357] [INSPIRE].

[105] J.P. Vary, T.J. Fields and H.-J. Pirner, Chiral perturbation theory in the Schwinger model, Phys. Rev. D 53 (1996) 7231 [INSPIRE].

[106] Y. Hosotani and R. Rodriguez, Bosonized massive N flavor Schwinger model, J. Phys. A 31 (1998) 9925 [hep-th/9804205] [INSPIRE].

[107] E. Abdalla, M.C.B. Abdalla and K.D. Rothe, Two dimensional quantum field theory, World Scientific Publishing Company, Singapore (2001).

[108] S. Nagy, Massless fermions in multi-flavor QED(2), Phys. Rev. D 79 (2009) 045004 [arXiv:0805.2009] [INSPIRE].

[109] A.P. Balachandran and A.R. de Queiroz, Quantum gravity: mixed states from diffeomorphism anomalies, JHEP 11 (2011) 126 [arXiv:1109.5290] [INSPIRE].

[110] J. Kogut and L. Susskind, Quark confinement and the puzzle of the ninth axial-vector current, Phys. Rev. D 15 (1974) 3468.

[111] J.B. Kogut and L. Susskind, How to solve the $\eta \rightarrow 3 \pi$ problem by seizing the vacuum, Phys. Rev. D 11 (1975) 3594 [inSPIRE].

[112] J.B. Kogut and D.K. Sinclair, Quark confinement and the evasion of Goldstone's theorem in (1+1)-dimensions, Phys. Rev. D 12 (1975) 1742 [INSPIRE].

[113] S. Weinberg, Phenomenological Lagrangians, Physica A 96 (1979) 327 [INSPIRE]. 
[114] E. Witten, Current algebra theorems for the U(1) Goldstone boson, Nucl. Phys. B 156 (1979) 269 [INSPIRE].

[115] M. Gell-Mann, R.J. Oakes and B. Renner, Behavior of current divergences under $\mathrm{SU}(3) \times \mathrm{SU}(3)$, Phys. Rev. 175 (1968) 2195.

[116] S. Weinberg, The quantum theory of fields, Cambridge University Press, Cambridge U.K. (1995).

[117] Particle Data Group collaboration, Review of particle physics, Phys. Rev. D 98 (2018) 030001 [INSPIRE].

[118] R. Hagedorn, Statistical thermodynamics of strong interactions at high energies, Nuovo Cim. Suppl. 3 (1965) 147.

[119] STAR collaboration, Strange particle production in $p+p$ collisions at $\sqrt{s}=200 \mathrm{GeV}$, Phys. Rev. C 75 (2007) 064901 [nucl-ex/0607033] [InSPIRE].

[120] STAR collaboration, Systematic measurements of identified particle spectra in pp, $d^{+} A u$ and Au+Au collisions from STAR, Phys. Rev. C 79 (2009) 034909 [arXiv:0808.2041] [INSPIRE].

[121] PHENIX collaboration, Measurement of neutral mesons in $p+p$ collisions at $\sqrt{s}=200 \mathrm{GeV}$ and scaling properties of hadron production, Phys. Rev. D 83 (2011) 052004 [arXiv: 1005.3674] [INSPIRE]. 